\title{
COHOMOLOGY OF GROUP EXTENSIONS
}

\author{
BY \\ G. HOCHSCHILD AND J-P. SERRE
}

Introduction. Let $G$ be a group, $K$ an invariant subgroup of $G$. The purpose of this paper is to investigate the relations between the cohomology groups of $G, K$, and $G / K$. As in the case of fibre spaces, it turns out that such relations can be expressed by a spectral sequence whose term $E_{2}$ is $H(G / K, H(K))$ and whose term $E_{\infty}$ is the graduated group associated with $H(G)$. This problem was first studied by R. C. Lyndon in his thesis [12]. Lyndon's procedure was to replace the full cochain complex of $G$ by an equivalent bigraduated subcomplex (of "normal" cochains, in his sense). His main result (generalized from the case of a direct product to the case of an arbitrary group extension, according to his indications) is that the bigraduated group associated with $H(G)$ is isomorphic with a factor group of a subgroup of $H(G / K, H(K))$. His methods can also be applied to special situations, like those considered in our Chapter III, and can give essentially the same results.

We give here two different approaches to the problem.

In Chapter I we carry out the method sketched by one of us in [13]. This method is based on the Cartan-Leray spectral sequence, $[3 ; 1]$, and can be generalized to other algebraic situations, as will be shown in a forthcoming paper of Cartan-Eilenberg [2]. Since the details of the Cartan-Leray technique have not been published (other than in seminar notes of limited circulation), we develop them in Chapter I. The auxiliary theorems we need for this purpose are useful also in other connections.

In Chapter II, which is independent of Chapter I, we obtain a spectral sequence quite directly by filtering the group of cochains for $G$. This filtration leads to the same group $E_{2}=H(G / K, H(K)$ ) (although we do not know whether or not the succeeding terms are isomorphic to those of the first spectral sequence) and lends itself more readily to applications, because one can identify the maps which arise from it. This is not always the case with the first filtration, and it is for this reason that we have developed the direct method in spite of the somewhat lengthy computations which are needed for its proofs.

Chapter III gives some applications of the spectral sequence of Chapter II. Most of the results could be obtained in the same manner with the spectral sequence of Chapter I. A notable exception is the connection with the theory of simple algebras which we discuss in $\$ 5$.

Finally, let us remark that the methods and results of this paper can be transferred to Lie Algebras. We intend to take up this subject in a later paper.

Received by the editors March 22, 1952. 


\section{Chapter I. General Methods( $\left.{ }^{(}\right)$}

1. Notation and definitions. Let $\Pi$ be an arbitrary group, $A$ an abelian group on which $\Pi$ operates from the left. $A$ is called a $\Pi$-module, and the transform of an element $a \in A$ by an element $\sigma \in \Pi$ is denoted $\sigma \cdot a$. By definition, $\sigma \cdot 0=0, \sigma \cdot(a+b)=\sigma \cdot a+\sigma \cdot b, 1 \cdot a=a$, and $\sigma \cdot(\tau \cdot a)=(\sigma \tau) \cdot a$. We shall denote by $A^{\mathrm{II}}$ the subgroup of $A$ which consists of all $a \in A$ for which $\sigma \cdot a=a$, for all $\sigma \in \Pi$. A set $\left(a_{i}\right), i \in I$, of elements $a_{i} \in A$ is called a $\Pi$-basis if the group $A$ is a free abelian group, with the elements $\sigma \cdot a_{i}, \sigma \in \Pi, i \in I$, being all distinct and constituting a basis. $A$ is called $\Pi$-free if it possesses a $\Pi$-basis.

If $A$ and $B$ are two $\Pi$-modules, the group $C=\operatorname{Hom}(A, B)$ of all homomorphisms of $A$ into $B$ is given the structure of a $\Pi$-module by setting $(\sigma \cdot f)(a)=\sigma \cdot f\left(\sigma^{-1} \cdot a\right)$. The elements of $C^{\mathrm{I}}$ are then the $\Pi$-homomorphisms of $A$ into $B$. We shall write $C^{\mathrm{II}}=\operatorname{Hom}^{\mathrm{II}}(A, B)$.

Complexes. A chain (cochain) complex is a graduated abelian group $C$ $=\sum_{n=0}^{\infty} C_{n}$, with an endomorphism $d$ such that $d^{2}=0, d\left(C_{0}\right)=(0)$, and, for $n>0, d\left(C_{n}\right) \subset C_{n-1}\left(d\left(C_{n}\right) \subset C_{n+1}\right.$, for all $n \geqq 0$, respectively). This gives rise to homology (cohomology) groups of $C$ in the usual way.

An augmentation of the chain complex $C$ is a homomorphism $\epsilon$ of $C_{0}$ into the additive group $Z$ of the integers such that $\epsilon \circ d=0$. An augmented complex $(C, \epsilon)$ is said to be acyclic if its homology groups $H_{i}(C)$ are $(0)$ for $i>0$, and if $\epsilon$ induces an isomorphism of $H_{0}(C)$ onto $Z$.

If $C$ is a chain complex and $A$ an abelian group, the group $C^{*}$ $=\sum_{n=0}^{\infty} \operatorname{Hom}\left(C_{n}, A\right)$ will be regarded as a cochain complex with regard to the endomorphism $d^{*}$ which is defined by setting $\left(d^{*} f\right)(x)=f(d x)$. We shall usually denote this complex by Hom $(C, A)$, although this conflicts-strictly speaking-with the notation introduced previously.

II-complexes. A chain complex $C$ with the structure of a II-module such that $\sigma\left(C_{n}\right)=C_{n}, \sigma \circ d=d \circ \sigma$, and $\epsilon \circ \sigma=\epsilon$, for all $\sigma \in \Pi$, is called a $\Pi$-complex. If each $C_{n}$ is $\Pi$-free, the $\Pi$-complex $C$ is said to be $\Pi$-free. A cochain $\Pi$-complex is defined analogously.

The homology groups $H_{i}(C)$ of a $\Pi$-complex $C$ are $\Pi$-modules in the natural fashion. If $A$ is a $\Pi$-module, the cochain complex $\operatorname{Hom}(C, A)$ is also a $\Pi$-module, and $\operatorname{Hom}^{\Pi}(C, A)$ is a subcomplex of $\operatorname{Hom}(C, A)$.

\section{Cohomology groups of a group $\Pi$ in a $\Pi$-module.}

Proposition 1. Let $C$ be $a \Pi$-free and acyclic $\Pi$-complex, $A$ a $\Pi$-module. Then the cohomology groups $H^{n}\left(\operatorname{Hom}^{\Pi}(C, A)\right)$ depend only on $\Pi$ and $A$, not on $C$. They are called the nth cohomology groups of $I I$ in $A$, and denoted $H^{n}(\Pi, A)\left({ }^{2}\right)$.

(1) The contents of $\S \S 1,2,4,5,6$ are mostly extracted from expositions made by H. Cartan and S. Eilenberg in a seminar conducted in Paris during the academic year 1950-1951, We in. clude them here for the convenience of the reader.

(2) This proposition is valid also for other cohomology theories, cf. [2], 
Actually, one proves more than this.

(a) If $C$ is $\Pi$-free, and $C^{\prime}$ is acyclic, there exists a $\Pi$-homomorphism $\phi: C \rightarrow C^{\prime}$, such that $\phi\left(C_{n}\right) \subset C_{n}^{\prime}, \epsilon^{\prime} \circ \phi=\epsilon$, and $\phi \circ d=d^{\prime} \circ \phi$. Furthermore, if $\psi$ is any other such homomorphism, there exists a II-homomorphism $k: C \rightarrow C^{\prime}$ such that $k\left(C_{n}\right) \subset C_{n+1}^{\prime}$, and $\phi-\psi=d^{\prime} \circ k+k \circ d$.

From this, one deduces at once the following:

(b) If $\phi$ and $\psi$ are two II-homomorphisms satisfying the conditions laid down in (a), then the corresponding homomorphisms $\phi^{*}$ and $\psi^{*}$ of $\operatorname{Hom}^{\mathrm{I}}\left(C^{\prime}, A\right)$ into $\operatorname{Hom}^{\mathrm{I}}(C, A)$ induce the same homomorphism of $H^{n}\left(\operatorname{Hom}^{\Pi}\left(C^{\prime}, A\right)\right)$ into $H^{n}\left(\operatorname{Hom}^{\Pi}(C, A)\right)$, for each $n \geqq 0$.

(c) If $C$ and $C^{\prime}$ are both II-free and acyclic, the homomorphism $\phi$ of (a) induces an isomorphism of $H^{n}\left(\operatorname{Hom}^{\text {II }}\left(C^{\prime}, A\right)\right)$ onto $H^{n}\left(\operatorname{Hom}^{\text {II }}(C, A)\right)$, and this isomorphism does not depend on the particular choice of $\phi$. It is called the canonical isomorphism.

Finally, one proves:

(d) For any $\Pi$, there exists a $\Pi$-free acyclic $\Pi$-complex.

All these results are well known (see $[4 ; 10])$ and we shall confine ourselves to recalling the proof of (d):

Construction of a $\Pi$-free acyclic $\Pi$-complex. Let $E$ be a set on which $\Pi$ operates without fixed points, i.e., such that, if $\sigma \in \Pi$ and $e \in E, \sigma \cdot e=e$ only if $\sigma=1$. One may, for instance, take $E=\Pi$, with the left translations as operators. One defines a complex $C(E)=\sum_{n=0}^{\infty} C(E)_{n}$ as follows. $C(E)_{n}$ is taken to be the free abelian group with the elements $\left(e_{0}, \cdots, e_{n}\right) \in E^{n+1}$ constituting a basis. The boundary operator $d$ is defined by the formula $d\left(e_{0}, \cdots, e_{n}\right)$ $=\sum_{i=0}^{n}(-1)^{i}\left(e_{0}, \cdots, \hat{e}_{i}, \cdots, e_{n}\right)$, where the symbol 1 denotes that the argument below it is to be omitted. The augmentation is defined by $\epsilon\left(e_{0}\right)=1$. $\Pi$ operates on $C(E)$ according to: $\sigma \cdot\left(e_{0}, \cdots, e_{n}\right)=\left(\sigma \cdot e_{0}, \cdots, \sigma \cdot e_{n}\right)$, and one verifies immediately that one so obtains a $\Pi$-complex.

We have then $d\left(C(E)_{0}\right)=(0)$, while $d\left(C(E)_{1}\right)$ coincides with the kernel of $\epsilon$, whence it is clear that $\epsilon$ induces an isomorphism of $H_{0}(C(E))$ onto $Z$. If $n>0$, and $c \in C(E)_{n}$, let $c^{\prime}$ denote the element of $C(E)_{n+1}$ which is obtained from $c$ by replacing each $(n+1)$-tuple $\left(e_{0}, \cdots, e_{n}\right)$ occurring in $c$ with $\left(e, e_{0}, \cdots, e_{n}\right)$, where $e$ is a fixed element of $E$. Then it is immediate that, if $d c=0$, we have $d c^{\prime}=c$, and we have shown that $C(E)$ is acyclic. From the fact that $\Pi$ operates without fixed points on $E$, it follows that each $C(E)_{n}$ is $\Pi$-free. Thus, $C(E)$ is a $\Pi$-free acyclic $\Pi$-complex.

If $A$ is a II-module, the elements of $\operatorname{Hom}^{\Pi}\left(C(E)_{n}, A\right)$ are the functions defined on $E^{n+1}$ with values in $A$ which satisfy the conditions $f\left(\sigma \cdot e_{0} \cdots, \sigma \cdot e_{n}\right)$ $=\sigma \cdot f\left(e_{0}, \cdots, e_{n}\right), \sigma \in \Pi$. In particular, if $E=\Pi$, with the left translations as operators, one arrives at the usual definition of the groups $H^{n}(\Pi, A)$ by the so-called homogeneous cochains $f$, where $f\left(\sigma \sigma_{0}, \cdots, \sigma \sigma_{n}\right)=\sigma \cdot f\left(\sigma_{0}, \cdots, \sigma_{n}\right)$, the coboundary operator $d^{*}$ being given by the formula $\left(d^{*} f\right)\left(\sigma_{0}, \cdots, \sigma_{n+1}\right)$ $=\sum_{i=0}^{n+1}(-1)^{i} f\left(\sigma_{0}, \cdots, \hat{\sigma}_{i}, \cdots, \sigma_{n+1}\right)$. 
Finally, let us recall that if one associates with such a cochain the "nonhomogeneous" cochain $\bar{f}\left(\sigma_{1}, \cdots, \sigma_{n}\right)=f\left(1, \sigma_{1}, \sigma_{1} \sigma_{2}, \cdots, \sigma_{1} \cdots \sigma_{n}\right)$, one obtains the usual coboundary operator $\delta \bar{f}\left(\sigma_{1}, \cdots, \sigma_{n+1}\right)=\sigma_{1} \cdot \bar{f}\left(\sigma_{2}, \cdots, \sigma_{n+1}\right)$ $+\sum_{i=1}^{n}(-1)^{i} \bar{f}\left(\sigma_{1}, \cdots, \sigma_{i} \sigma_{i+1}, \cdots, \sigma_{n+1}\right)+(-1)^{n+1} \bar{f}\left(\sigma_{1}, \cdots, \sigma_{n}\right)$.

Proposition 2. Let $E$ and $E^{\prime}$ be two sets on which $\Pi$ operates without fixed points, and let $\rho$ be a mapping of $E$ into $E^{\prime}$ which commutes with the $\Pi$-operators. Then, for each $n \geqq 0, \rho$ induces on $H^{n}\left(\operatorname{Hom}^{\Pi}\left(C\left(E^{\prime}\right), A\right)\right)$ the canonical isomorphism onto $H^{n}\left(\operatorname{Hom}^{\Pi}(C(E), A)\right)$.

In fact, it is evident that $\rho$ induces a homomorphism $\phi$ of $C(E)$ into $C\left(E^{\prime}\right)$ which satisfies the conditions of (a) above; and the result follows at once from (c).

Let us apply this to the case where $E=E^{\prime}=\Pi$, with the left translations as operators, and let us put $\rho(e)=e \sigma$, where $\sigma$ is a fixed element of $\Pi$. Then $\rho$ evidently commutes with the left translations and hence induces the canonical isomorphism of $H^{n}(\Pi, A)$ onto itself, which is the identity map. Hence we have:

Corollary. Let $\Pi$ be a group, $A$ a $\Pi$-module, $\sigma \in \Pi$. For each homogeneous cochain $f$ let us define the homogeneous cochain $M_{\sigma} f$ by $\left(M_{\sigma} f\right)\left(\sigma_{0}, \cdots, \sigma_{n}\right)$ $=f\left(\sigma_{0} \sigma, \cdots, \sigma_{n} \sigma\right)$. Then the map $M_{\sigma}$ commutes with the coboundary and induces the identity map on $H^{n}(\Pi, A)$.

Translated into the nonhomogeneous cohomology theory, this means that, if $\bar{f}$ is a nonhomogeneous $n$-cocycle, the cocycle whose value for $\sigma_{1}, \cdots, \sigma_{n}$ is $\sigma \cdot \bar{f}\left(\sigma^{-1} \sigma_{1} \sigma, \cdots, \sigma^{-1} \sigma_{n} \sigma\right)$ is cohomologous to $\bar{f}\left({ }^{3}\right)$.

3. Applications. Let $G$ be a group, $K$ a subgroup of $G$. Let $K$ operate on $G$ by multiplication on the left. We can apply the results of $\$ 2$ with $E=G$ and $\Pi=K$, introducing the cochain complex $B=\operatorname{Hom}^{K}(C(G), A)$, where $A$ is an arbitrary $K$-module. A homogeneous element of degree $n$ of $B$ is a function $f$ defined on $G^{n+1}$, with values in $A$, and such that $f\left(\sigma \gamma_{0}, \cdots, \sigma \gamma_{n}\right)$ $=\sigma \cdot f\left(\gamma_{0}, \cdots, \gamma_{n}\right)$, for $\sigma \in K$ and $\gamma_{i} \in G$.

Let $C(K, A)$ be the complex of the homogeneous cochains for $K$ in $A$. The injection $\rho: K \rightarrow G$ gives rise to the dual homomorphism $\rho^{*}$ of $B$ into $C(K, A)$ which is simply the map obtained by restricting the arguments to $K$. Applying Proposition 2 to $\rho$, we obtain:

Proposition 3. Let $G$ be a group, $K$ a subgroup of $G, A$ a $K$-module, $B=\operatorname{Hom}^{K}(C(G), A)$. Then the homomorphism of $B$ into $C(K, A)$ which maps every cochain $f \in B$ into its restriction to $K$ induces an isomorphism of $H^{n}(B)$ onto $H^{n}(K, A)$, for all $n \geqq 0$.

It is easy to define the inverse isomorphism of the above directly. In fact, by Proposition 2, it suffices to take the homomorphism which is induced by

(3) This result is well known, cf. $[12, \$ 10]$ and Theorem 1.3 of [11] (for dimension 2). 
any map $\psi$ of $G$ into $K$ for which $\psi(\sigma \gamma)=\sigma \psi(\gamma)$, for all $\sigma \in K$ and $\gamma \in G$.

Corollary $\left.{ }^{4}\right)$. Let $B_{0}$ be the group of the maps $f$ of $G$ into the $K$-module $A$ such that $f(\sigma \gamma)=\sigma \cdot f(\gamma)$, for all $\sigma \in K, \gamma \in G$. Let $G$ operate on $B_{0}$ according to the definition $\left(\gamma_{1} \cdot f\right)(\gamma)=f\left(\gamma \gamma_{1}\right)$. Let $\phi$ be the $K$-homomorphism of $B_{0}$ into $A$ defined by $\phi(f)=f(1)$. Then the restriction of the arguments from $G$ to $K$, combined with the homomorphism $\phi$, induces an isomorphism of $H^{n}\left(G, B_{0}\right)$ onto $H^{n}(K, A)$, for all $n \geqq 0$.

Let $B$ denote the group of Proposition 3. If $f$ is a homogeneous element of degree $n$ of $B$, let us define $\alpha(f) \in C^{n}\left(G, B_{0}\right)$ by setting $\alpha(f)\left(\gamma_{0}, \cdots, \gamma_{n}\right)(\gamma)$ $=f\left(\gamma \gamma_{0}, \cdots, \gamma \gamma_{n}\right)$. Clearly, $\alpha$ commutes with the coboundary operator. Furthermore, $\alpha$ is an isomorphism onto: for $h \in C^{n}\left(G, B_{0}\right)$, $\alpha^{-1}(h)\left(\gamma_{0}, \cdots, \gamma_{n}\right)=h\left(\gamma_{0}, \cdots, \gamma_{n}\right)(1)$. Hence $\alpha^{-1}$ induces an isomorphism of $H^{n}\left(G, B_{0}\right)$ onto $H^{n}(B)$. If this is combined with the isomorphism of Proposition 3, one obtains an isomorphism of $H^{n}\left(G, B_{0}\right)$ onto $H^{n}(K, A)$, and one sees immediately from the definitions of $\alpha^{-1}$ and $\phi$ that this is the isomorphism described in the corollary.

Remark. If the $K$-operators on $A$ can be extended so that $A$ becomes a $G$-module, $B_{0}$ may be identified with the group $F$ of all maps of the set $G / K$ of the left cosets $K \gamma$ into $A$, made into a $G$-module by setting, for $g \in F$, $\gamma \in G$, and $x \in G / K,(\gamma \cdot g)(x)=\gamma \cdot g(x \gamma)$. In fact, if $f \in B_{0}$, we define $\bar{f} \in F$ by setting $\bar{f}(K \gamma)=\gamma^{-1} f(\gamma)$, and the map $f \rightarrow \bar{f}$ is a $G$-isomorphism of $B_{0}$ onto $F$.

4. A preliminary result. Let $\Pi$ be a group, $U=\sum_{j=0}^{\infty} U_{j}$ a cochain $\Pi$-complex. Put $L^{p, q}=C^{p}\left(\Pi, U_{q}\right)$, the group of nonhomogeneous $p$-cochains of $\Pi$ in $U_{q}$. Let $C(\Pi, U)=\sum_{p, q} L^{p, q}$. Thus, $C(\Pi, U)$ is a bigraduated group, on which we define two coboundary operators, as follows: $d_{\Pi}: L^{p, q} \rightarrow L^{p+1, q}$ is the usual nonhomogeneous coboundary operator on $p$-cochains, as given in $\$ 2$, just preceding Proposition 2. The other coboundary operator $d_{U}: L^{p, q}$ $\rightarrow L^{p, q+1}$ is defined by setting $\left(d_{U} f\right)\left(\sigma_{1}, \cdots, \sigma_{p}\right)=d\left(f\left(\sigma_{1}, \cdots, \sigma_{p}\right)\right)$, where $d$ denotes the coboundary operator in $U$, and $\sigma_{i} \in \Pi$.

We have $L^{0, q}=U_{q}$, so that $U$ is a subgroup of $C(\Pi, U)$. From the two operators $d_{\mathrm{\Pi}}$ and $d_{U}$, we define a third coboundary operator $d=d_{\Pi}+(-1)^{p} d_{U}$ : $L^{p, q} \rightarrow L^{p+1, q}+L^{p, q+1}$. With this new operator $d, C(\Pi, U)$ constitutes a cochain complex, and since $d_{\mathrm{I}}=0$ on $U^{\mathrm{I}}$, the restriction to $U^{\mathrm{II}}$ of the coboundary operator $d$ coincides with $d_{U}$.

Proposition 4. Suppose that $H^{n}\left(\Pi, U_{j}\right)=(0)$, for all $j \geqq 0$ and all $n>0$. Then the injection of $U^{\Pi}$ into $C(\Pi, U)$ defines an isomorphism of $H^{n}\left(U^{\Pi}\right)$ onto $H^{n}(C(\Pi, U))$, for all $n \geqq 0$.

Put $A^{i}=\sum_{q \geqq i} \sum_{p=0}^{\infty} L^{p, q}, B^{i}=A^{i} \cap U^{\mathrm{I}}=\sum_{q \geqq i} U_{\mathfrak{a}}^{\mathrm{II}}$. It will suffice to

(4) This result is due to A. Weil (Sur la théorie du corps de classes, Jour. Math. Soc. Jap. vol. 3 (1951) pp. 1-35, footnote 4). For a direct proof see G. Hochschild and T. Nakayama (Cohomology in class field theory, Ann. of Math. vol. 55 (1952) Lemma 1.1). 
prove that the canonical homomorphism $B^{i} / B^{i+1} \rightarrow A^{i} / A^{i+1}$ induces an isomorphism of $H^{n}\left(B^{i} / B^{i+1}\right)$ onto $H^{n}\left(A^{i} / A^{i+1}\right)$, for each $n \geqq 0$. In fact, if this is proved an application of the "five lemma" $\left(^{5}\right)$ to the exact sequences for the triples $\left(A^{i}, A^{i+p}, A^{i+p+1}\right)$ and $\left(B^{i}, B^{i+p}, B^{i+p+1}\right)$ shows, by induction on $p$, that the canonical homomorphism $H^{n}\left(B^{i} / B^{i+p}\right) \rightarrow H^{n}\left(A^{i} / A^{i+p}\right)$ is an isomorphism onto for each $p>0$. Proposition 4 then follows by taking $i=0$ and $p=n+2$.

Now $A^{i} / A^{i+1}$ is isomorphic with $\sum_{p=0}^{\infty} L^{p, i}=C\left(\Pi, U_{i}\right)$, with the ordinary coboundary operator for nonhomogeneous $p$-cochains. The homomorphism $B^{i} / B^{i+1} \rightarrow A^{i} / A^{i+1}$ corresponds simply to the injection of $U_{i}^{\mathrm{II}}$ into $C^{0}\left(\Pi, U_{i}\right)$ $=U_{i}$, and therefore the statement that it induces an isomorphism of the cohomology groups is equivalent to our assumption that $H^{n}\left(\Pi, U_{i}\right)=(0)$, for $n>0$.

5. The spectral sequence of Cartan-Leray. Let $C(\Pi, U)$ be the bigraduated complex defined in $\$ 4$. We shall define a filtration on this complex and then determine the groups $E_{1}$ and $E_{2}$ of the corresponding spectral sequence $\left.{ }^{6}\right)$.

Definition of the filtration. Let $L_{i}^{q}=\sum_{p \geqq i} L^{p, q}$, and $L_{i}=\sum_{q=0}^{\infty} L_{i}^{q}$. Evidently, $C(\Pi, U)=L_{0} \supset L_{1} \supset \cdots, d\left(L_{i}\right) \subset L_{i}$, and $C^{p}\left(\Pi, U_{q}\right) \cap L_{i}=(0)$, if $i>p$. Thus the groups $L_{i}$ define a filtration of $C(\Pi, U)$.

Calculation of $E_{1}$. By definition, $E_{1}^{p, q}=H^{p+q}\left(L_{p} / L_{p+1}\right)$. In our case, $L_{p} / L_{p+1}$, with the coboundary operator induced by $d$, is isomorphic with $\sum_{q=0}^{\infty} L^{p, q}=C^{p}(\Pi, U)$, with the coboundary operator $(-1)^{p} d_{U}$. Hence we have:

Lemma 1. The term $E_{1}^{p, q}$ of the spectral sequence is canonically isomorphic with $C^{p}\left(\Pi, H^{q}(U)\right)$.

Calculation of $E_{2}$. Let us recall that the differential operator $d_{1}$ on $E_{1}$ $=\sum_{p, q} E_{1}^{p, q}$ maps $E_{1}^{p, q}$ into $E_{1}^{p+1, q}$, by the coboundary map of the exact sequence for the triple $\left(L_{p}, L_{p+1}, L_{p+2}\right)$ which sends $H^{p+q}\left(L_{p} / L_{p+1}\right)$ into $H^{p+q+1}\left(L_{p+1} / L_{p+2}\right)$. The term $E_{2}^{p, q}$ is the $(p, q)$-cohomology group in the bigraduated complex $E_{1}$ (with respect to the operator $d_{1}$ ). We claim that, under the isomorphism of Lemma $1, d_{1}$ is transformed into the coboundary operator for the cochains of $\Pi$ in the $\Pi$-module $H^{q}(U)$.

In order to see this, let $f \in C^{p}\left(\Pi, H^{q}(U)\right)$, and let us compute $d_{1} f$. For this, we must first choose an element $x \in L_{p}$ which is a cocycle $\bmod L_{p+1}$ and whose cohomology class is $f$. If $\sigma_{1}, \cdots, \sigma_{p}$ are elements of $\Pi$, let $x\left(\sigma_{1}, \cdots, \sigma_{p}\right)$ be a cocycle in $U_{q}$ whose cohomology class is $f\left(\sigma_{1}, \cdots, \sigma_{p}\right)$. We have then

(5) We recall the "five lemma": suppose we have two exact sequences of five terms each and five homomorphisms of the groups of the first sequence into the corresponding groups of the second, such that the commutativity relations hold in the resulting diagram. Then, if the four extreme homomorphisms are isomorphisms onto, so is the middle one.

${ }^{(6)}$ For the notation and the definitions relating to spectral sequences we refer the reader to $[14$, Chapter I, no. 5] (see also below, Chapter III, $\$ \$ 1,3$ ). However, we shall omit the signs *, since no confusion with homology can arise here. 
$d x=d_{\Pi} x+(-1)^{p} d_{U} x=d_{\Pi} x \in L^{p+1, q}$. If this is written out according to the coboundary formula for $d_{\Pi}$, it is evident that $d x\left(\sigma_{1}, \cdots, \sigma_{p+1}\right)$ is a cocycle in $U_{q}$, for all $\sigma_{i} \in \Pi$. Hence $d x$ defines an element $y \in E_{1}^{p+1, q}$, and by the definition of $d_{1}$ we have $d_{1} f=y$. Clearly, $y$ is the coboundary of $f$, regarded as a cochain for $\Pi$ in $H^{q}(U)$. Hence we have:

Lemma 2. The term $E_{2}^{p, q}$ of the spectral sequence derived from the filtration $\left(L_{i}\right)$ is canonically isomorphic with $H^{p}\left(\Pi, H^{q}(U)\right)$.

The term $E_{\infty}$. As in every spectral sequence, the group $E_{\infty}$ is isomorphic with the graduated group associated with $H(C(\Pi, U))$, filtered by the subgroups arising from the $L_{i}$. (We recall that if $A$ is any additive group, filtered by a nonincreasing sequence of subgroups $A_{i}$, the associated graduated group is defined as the graduated group whose component of degree $i$ is $A_{i} / A_{i+1}$. If $A$ is also graduated, compatibly with the filtration, the associated group is bigraduated in the natural fashion.) If we combine the above result with Proposition 4, we obtain the following result of Cartan-Leray [3], [1]:

Proposition 5. Let $U$ be a cochain $\Pi$-complex, such that the groups $H^{i}\left(\Pi, U_{j}\right)$ vanish for all $j \geqq 0$ and all $i>0$, where $U_{j}$ denotes the subgroup of $U$ consisting of the homogeneous elements of degree $j$. Then, in the spectral sequence $\left(E_{r}\right)$ which is derived from the filtration $\left(L_{i}\right)$, the term $E_{2}^{p, q}$ is isomorphic with $H^{p}\left(\Pi, H^{q}(U)\right)$, and $E_{\infty}$ is isomorphic with the graduated group associated with $H\left(U^{\mathrm{I}}\right)$, filtered by the subgroups arising from the $L_{i}$.

6. The vanishing of certain cohomology groups. Let $A$ be a $\Pi$-module. By a mean on $A$ we shall understand an additive function $I$ which associates with each map $f: \Pi \rightarrow A$ an element $I(f) \in A$, such that:

(a) If $f(\sigma)=a \in A$, for each $\sigma \in \Pi$, then $I(f)=a$.

(b) For all $\sigma \in \Pi, I(\sigma \cdot f)=\sigma \cdot I(f)$, where $(\sigma \cdot f)(\tau)=\sigma \cdot f\left(\sigma^{-1} \tau\right)$.

Proposition 6. If $A$ is a $\Pi$-module which admits a mean, then $H^{n}(\Pi, A)$ $=(0)$, for all $n>0$.

In fact, let $f$ be a homogeneous $n$-cocycle for $\Pi$ in $A$. For fixed $\sigma_{1}, \cdots, \sigma_{n}$ in $\Pi$, the map $\sigma \rightarrow f\left(\sigma, \sigma_{1}, \cdots, \sigma_{n}\right)$ has a mean value $\left(I_{n} f\right)\left(\sigma_{1}, \cdots, \sigma_{n}\right) \in A$. It is immediate that $\left(I_{n} f\right)\left(\sigma \sigma_{1}, \cdots, \sigma \sigma_{n}\right)=\sigma \cdot\left(I_{n} f\right)\left(\sigma_{1} \cdots, \sigma_{n}\right)$. Thus, $I_{n} f$ is a homogeneous $(n-1)$-cochain for $\Pi$ in $A$, and it is easy to verify that $d\left(I_{n} f\right)$ $=f$.

Corollary $\left({ }^{7}\right)$. Let $L$ be a $\Pi$-free $\Pi$-module, $B$ an arbitrary $\Pi$-module, $A=\operatorname{Hom}(L, B)$. Then $H^{n}(\Pi, A)=(0)$, for all $n>0$.

Decomposing $L$ into a direct sum, one sees that it suffices to prove the corollary in the case where $L$ has a $\Pi$-basis consisting of a single element.

(7) Cf. R. C. Lyndon, Cohomology theory of groups with a single defining relation, Ann. of Math. vol. 52 (1950) p. 653, Theorem 2.2. 
In this case, $A$ is isomorphic with the $\Pi$-module of all maps $\phi: \Pi \rightarrow B$, where $(\sigma \cdot \phi)(\tau)=\sigma \cdot \phi\left(\sigma^{-1} \tau\right)$, for $\sigma, \tau \in \Pi$. A map $f: \Pi \rightarrow A$ may then be regarded as a map $f^{\prime}: \Pi \times \Pi \rightarrow A$, and one obtains a mean on $A$ by setting $I(f)(\sigma)=f^{\prime}(\sigma, \sigma)$.

(Actually, this corollary could easily be proved directly; it can also be obtained as a consequence of the corollary to Proposition 3.)

Remarks. 1. Proposition 6 covers a number of the known cases $\left({ }^{8}\right)$ in which the cohomology groups vanish; for instance, the case where $\Pi$ is finite of order $m$ and every element of $A$ is uniquely divisible by $m$, or the case where $\Pi$ is compact and where one deals with continuous cochains for $\Pi$ in a vector group $R^{n}$ (cf. K. Iwasawa, On some types of topological groups, Ann. of Math. vol. 50 (1949) pp. 507-558).

2. The corollary to Proposition 6 shows that whenever the complex $U$, dealt with in $\$ 5$, is of the form Hom $(C, A)$, where $C$ is a $\Pi$-free chain complex, one can apply Proposition 5 to $U$. For instance, one could take for $C$ the singular complex of a space on which $\Pi$ operates without fixed points; $c f$. $[4 ; 6]$.

7. The spectral sequence for group extensions. Let $G$ be a group, $K$ an invariant subgroup of $G, A$ a $G$-module. Let $M$ denote the complex Hom $(C(G), A)$, where the notation is that of $\S \S 2,3$. The elements of degree $n$ of $M$ are the functions $f: G^{n+1} \rightarrow A$, the coboundary operator, $d$, being defined by $(d f)\left(\gamma_{0}, \cdots, \gamma_{n}\right)=\sum_{i=0}^{n}(-1)^{i} f\left(\gamma_{0}, \cdots, \hat{\gamma}_{i}, \cdots, \gamma_{n}\right)$.

Consider the subcomplex $M^{K}$ of $M$. Since $K$ is invariant in $G, G / K$ operates canonically on $M^{K}$. Furthermore, $M^{K}$, regarded as a $G / K$-module, admits a mean, in the sense of $\S 6$. In fact, let $f$ be a function on $G / K$ with values in the homogeneous component of degree $n$ of $M^{K}$. We set, for $\gamma_{0}, \cdots, \gamma_{n} \in G, I(f)\left(\gamma_{0}, \cdots, \gamma_{n}\right)=f\left(\bar{\gamma}_{0}\right)\left(\gamma_{0}, \cdots, \gamma_{n}\right)$, where $\bar{\gamma}_{0}$ denotes the canonical image of $\gamma_{0}$ in $G / K$. Then $I(f)$ is a homogeneous element of degree $n$ in $M^{K}$, and one sees immediately that $I$ is a mean. Hence we can apply Proposition 5 with $\Pi=G / K$, and $U=M^{K}$. We have then $U^{\Pi}=M^{G}=\operatorname{Hom}^{G}(C(G), A)$, so that $H^{n}\left(U^{\mathrm{II}}\right)=H^{n}(G, A)$. On the other hand, Proposition 3 shows that $H^{n}(U)$ $=H^{n}\left(M^{K}\right)$ is canonically isomorphic with $H^{n}(K, A)$. Hence Proposition 5 yields the following:

Proposition 7. Let $G$ be a group, $K$ an invariant subgroup of $G, A$ a $G$-module. Then there exists a spectral sequence $\left(E_{r}\right)$ in which the term $E_{2}^{p, a}$ is isomorphic with $H^{p}\left(G / K, H^{q}(K, A)\right)$, and $E_{\infty}$ is isomorphic with the graduated group associated with $H(G, A)$, appropriately filtered.

We can describe the $G / K$-operators on $H(K, A)$ quite explicitly: If $f$ is a $q$-cochain for $K$ in $A$, and $\gamma \in G$, let $(\gamma \cdot f)\left(\sigma_{0}, \cdots, \sigma_{n}\right)=$ $\boldsymbol{\gamma} \cdot f\left(\gamma^{-1} \sigma_{0} \gamma, \cdots, \gamma^{-1} \sigma_{n} \gamma\right)$. Then the map $f \rightarrow \gamma \cdot f$ induces an automorphism $M_{\gamma}$ of $H^{q}(K, A)$. By the corollary to Proposition 2, $M_{\gamma}$ depends only on the

(8) For instance, if there are defined on $A$ a topology and an operation " $\lambda$ " (in the sense of [1], 2d note, no. 4), $A$ has the mean: $I(f)=\sum_{\sigma \in \Pi} \sigma \circ \lambda \circ \sigma^{-1} f(\sigma)$. 
canonical image $\bar{\gamma}$ of $\gamma$ in $G / K$, and one verifies that it is the automorphism which corresponds to $\bar{\gamma}$ in the above.

In order to keep our exposition within reasonable bounds we have confined ourselves to cohomology throughout. Actually, the results of this chapter can be transcribed into homology without difficulty. One must merely replace the operation "Hom" by the operation " $\otimes$ " of taking the tensor product of a right module by a left module, and the passage $A \rightarrow A^{\mathrm{I}}$ by the passage $A \rightarrow A_{\mathrm{I}}$, where $A_{\mathrm{I}}$ denotes the factor group of $A$ by the subgroup generated by the elements of the form $a-\sigma \cdot a$, with $a \in A$ and $\sigma \in \Pi$, cf. [2].

For the reasons we have explained in the introduction, we pursue the study of the spectral sequence of Proposition 7 no further. The reader may convince himself that one can obtain the results of Chapter III (except for the interpretation of the transgression) from Proposition 7.

\section{Chapter II. The direct method}

1. Filtrations. Let $G$ be a group, $M$ a $G$-module. Write $A^{n}=C^{n}(G, M)$, the group of "normalized" $n$-cochains for $G$ in $M$, i.e., of the functions $f: G^{n} \rightarrow M$, such that $f\left(\gamma_{1}, \cdots, \gamma_{n}\right)=0$ whenever one of the $\gamma_{i}$ is equal to 1 . By definition, $A^{0}=C^{0}(G, M)=M$. Let $A=\sum_{n=0}^{\infty} A^{n}$. Thus, $A$ is a graduated group. We denote by $d$ the nonhomogeneous coboundary operator:

$$
\begin{aligned}
(d f)\left(\gamma_{1}, \cdots, \gamma_{n+1}\right)= & \gamma_{1} \cdot f\left(\gamma_{2}, \cdots, \gamma_{n+1}\right) \\
& +\sum_{i=1}^{n}(-1)^{i} f\left(\gamma_{1}, \cdots, \gamma_{i} \gamma_{i+1}, \cdots, \gamma_{n+1}\right) \\
& +(-1)^{n+1} f\left(\gamma_{1}, \cdots, \gamma_{n}\right) .
\end{aligned}
$$

It is easily seen that, if $f$ is normalized, so is $d f$, so that $d\left(A^{n}\right) \subset A^{n+1}$. As is well known, normalization does not influence cohomology, and we have $H^{n}(A)$ $=H^{n}(G, M)$.

Let $K$ be a subgroup of $G$. We define a filtration $\left(A_{j}\right)$ of $A$ as follows: $A_{j}=A$, for $j \leqq 0$. For $j>0$, we set $A_{j}=\sum_{n=0}^{\infty} A_{j} \cap A^{n}$, where $A_{j} \cap A^{n}=(0)$, if $j>n$, and where, for $j \leqq n, A_{j} \cap A^{n}$ is the group of all elements $f \in A^{n}$ for which $f\left(\gamma_{1}, \cdots, \gamma_{n}\right)=0$ whenever $n-j+1$ of the arguments belong to the subgroup $K$. Evidently, $d\left(A_{j}\right) \subset A_{j}$, so that the groups $A_{j}$ constitute a filtration.

Paired modules. Let $M, N$, and $P$ be three $G$-modules. A pairing of $M$ and $N$ to $P$ is a map $M \times N \rightarrow P ;(m, n) \rightarrow m \cup n$, such that $\left(m_{1}-m_{2}\right) \cup n$ $=m_{1} \cup n-m_{2} \cup n, \quad m \cup\left(n_{1}-n_{2}\right)=m \cup n_{1}-m \cup n_{2}, \quad$ and $\gamma \cdot(m \cup n)=(\gamma \cdot m)$ $\cup(\gamma \cdot n)$. The cup product of cochains is a pairing of $C(G, M)$ and $C(G, N)$ to $C(G, P)$ such that $C^{p}(G, M) \cup C^{q}(G, N) \subset C^{p+q}(G, P)$ whose explicit definition is:

$$
(f \cup g)\left(\gamma_{1}, \cdots, \gamma_{p+q}\right)=f\left(\gamma_{1}, \cdots, \gamma_{p}\right) \cup \gamma_{1} \cdots \gamma_{p} \cdot g\left(\gamma_{p+1}, \cdots, \gamma_{p+q}\right) .
$$


One has then $d(f \cup g)=(d f) \cup g+(-1)^{p} f \cup(d g)$, whence it is clear that the cup product also induces a pairing of $H^{p}(G, M)$ and $H^{q}(G, N)$ to $H^{p+q}(G, P)$. The above filtration is compatible with pairing by cup products, in the sense that if $A_{j}, B_{j}, C_{j}$ denote the groups of the filtrations for $M, N, P$, respectively, we have $A_{r} \cup B_{s} \subset C_{r+s}$, and we then have induced pairings of the groups of the spectral sequences, such that $E_{t}^{j, 1}(A) \cup E_{t}^{j^{\prime}, i^{\prime}}(B) \subset E_{t}^{j+j^{\prime}, i+t^{\prime}}(C)$.

In the case where $K$ is invariant in $G$, we can introduce a second filtration $\left(A_{j}^{*}\right)$ of $A$ which has the defect of not being compatible with cup products but which will be very helpful in the computation of the spectral sequence. We again define $A_{j}^{*}=A, j \leqq 0$. For $j>0$, we set $A_{j}^{*}=\sum_{n=0}^{\infty} A_{j}^{*} \cap A^{n}$, where, for $j \leqq n, A_{j}^{*} \cap A^{n}$ is defined as the group of all $f \in A^{n}$ for which $f\left(\gamma_{1}, \cdots, \gamma_{n}\right)$ depends only on $\gamma_{1}, \cdots, \gamma_{n-j}$ and the cosets $\gamma_{n-j+1} K, \cdots$, $\gamma_{n} K$, while $A_{j}^{*} \cap A^{n}=(0)$, for $j>n$. Evidently, we have again $d\left(A_{j}^{*}\right) \subset A_{j}^{*}$. Furthermore, it is clear that $A_{j}^{*} \subset A_{j}$, for all $j$.

Proposition 1. If $E_{r}, E_{r}^{*}$ denote the groups of the spectral sequences derived from the filtrations $\left(A_{j}\right),\left(A_{j}^{*}\right)$, respectively, then the injections $A_{j}^{*} \rightarrow A_{j}$ induce isomorphisms of $E_{r}^{*}$ onto $E_{r}$, for each $r \geqq 1$.

This will follow trivially as soon as we have proved it for the case $r=1$. Hence it will suffice to prove that the injections $A_{j}^{*} \rightarrow A_{j}$ induce isomorphisms of $H\left(A_{j}^{*} / A_{j+1}^{*}\right)$ onto $H\left(A_{j} / A_{j+1}\right)$, for all $j$. If we apply the "five lemma" to the exact sequences for the pairs $\left(A_{j}, A_{j+1}\right)$ and $\left(A_{j}^{*}, A_{j+1}^{*}\right)$, we see that this will follow if we prove that the induced maps $H^{n}\left(A_{j}^{*}\right) \rightarrow H^{n}\left(A_{j}\right)$ are isomorphisms onto, for all $n$ and $j$. From the exact sequence for the pair $\left(A_{j}, A_{j}^{*}\right)$, it is clear that this will be the case provided that the following lemma holds:

\section{Lemma 1. $H^{n}\left(A_{j} / A_{j}^{*}\right)=(0)$, for all $n$ and $j$.}

We have to show the following. If $f \in A_{j} \cap A^{n}$ and $d f \in A_{j}^{*}$, then there is an element $g \in A_{j}$ such that $f-d g \in A_{j}^{*}$. This holds trivially for $j \leqq 0$ and for $j>n$, so that we may suppose that $0<j \leqq n$. Now consider the case $j=n$. Then $f\left(\gamma_{1}, \cdots, \gamma_{n}\right)=0$ whenever one of the $\gamma_{i}$ belongs to $K$, and $d f\left(\gamma_{1}, \cdots, \gamma_{n+1}\right)$ depends only on $\gamma_{1}$ and the cosets $\gamma_{i} K$ for $i>1$. From these facts and the coboundary formula, applied to $d f\left(\gamma_{1}, \cdots, \gamma_{i}, \sigma, \gamma_{i+1}, \cdots, \gamma_{n}\right)$ $=0$, it follows at once that, for $\sigma \in K, f\left(\gamma_{1}, \cdots, \gamma_{i} \sigma, \gamma_{i+1}, \cdots, \gamma_{n}\right)$ $=f\left(\gamma_{1}, \cdots, \gamma_{i}, \quad \sigma \gamma_{i+1}, \cdots, \gamma_{n}\right), \quad$ if $1 \leqq i<n$, and $f\left(\gamma_{1}, \cdots, \gamma_{n} \sigma\right)$ $=f\left(\gamma_{1}, \cdots, \gamma_{n}\right)$, whence $f \in A_{n}^{*}$. Hence we may now suppose that $0<j<n$, and it will clearly suffice to prove the following. Let $0 \leqq i<j<n, f \in$ $A_{j} \cap A_{i}^{*} \cap A^{n}$, and $d f \in A_{j}^{*}$. Then there is an element $g \in A_{j}$ such that $f-d g$ $\in A_{j} \cap A_{i+1}^{*}$. We shall proceed to construct such an element $g$ by successively defining $g_{j}, g_{j+1}, \cdots, g_{n}=g$ so as to satisfy increasingly stringent conditions.

If the $n-j+1$ arguments $\sigma_{j-i}, \cdots, \sigma_{n-i}$ are in $K$, we have, since $f \in$ $A_{j} \cap A^{n}, f\left(\gamma_{1}, \cdots, \gamma_{j-i-1}, \sigma_{j-i}, \cdots, \sigma_{n-i}, \gamma_{n-i+1}, \cdots, \gamma_{n}\right)=0$. Let $g_{j}=0$, and suppose then that we have already found an element $g_{p} \in A_{j} \cap A_{\mathfrak{i}}^{*} \cap A^{n-1}$, 
$j \leqq p<n$, such that $\left(f-d g_{p}\right)\left(\gamma_{1}, \cdots, \gamma_{p-i-1}, \sigma_{p-i}, \cdots, \sigma_{n-i}, \gamma_{n-i+1}, \cdots, \gamma_{n}\right)$ $=0$, for all $\gamma_{r}$ in $G$ and all $\sigma_{s}$ in $K$. Write $f_{p}=f-d g_{p}$, choose representatives $x^{*}$ in $G$ for the cosets $x=x^{*} K$, taking $K^{*}=1$, and define, for $\sigma \in K$, and $\gamma_{r} \in G$,

$$
\begin{aligned}
h_{p}\left(\gamma_{1}, \cdots, \gamma_{p-i-1}, x^{*} \sigma, \gamma_{p-i+1},\right. & \left.\cdots, \gamma_{n}\right) \\
& =f_{p}\left(\gamma_{1}, \cdots, \gamma_{p-i-1}, x^{*}, \sigma, \gamma_{p-i+1}, \cdots, \gamma_{n}\right) .
\end{aligned}
$$

Then $h_{p} \in A_{j} \cap A_{i}^{*} \cap A^{n-1}$. Now consider the value

$$
d h_{p}\left(\gamma_{1}, \cdots, \gamma_{p-i-1}, x^{*} \sigma_{p-i}, \sigma_{p+1-i}, \cdots, \sigma_{n-i}, \gamma_{n-i+1}, \cdots, \gamma_{n}\right) .
$$

If it is written out according to the coboundary formula, and if the values of $h_{p}$ are written as values of $f_{p}$, we find that the first nonzero term is $(-1)^{p-i} h_{p}\left(\gamma_{1}, \cdots, \gamma_{p-i-1}, x^{*} \sigma_{p-i} \sigma_{p+1-i}, \cdots, \sigma_{n-i}, \gamma_{n-i+1}, \cdots, \gamma_{n}\right)$ $=(-1)^{p-i} f_{p}\left(\gamma_{1}, \cdots, \gamma_{p-i-1}, x^{*}, \sigma_{p-i} \sigma_{p+1-i}, \cdots, \sigma_{n-i}, \gamma_{n-i+1}, \cdots, \gamma_{n}\right)$. On the other hand, if we write out the coboundary

$$
d f_{p}\left(\gamma_{1}, \cdots, \gamma_{p-i-1}, x^{*}, \sigma_{p-i}, \sigma_{p+1-i}, \cdots, \sigma_{n-i}, \gamma_{n-i+1}, \cdots, \gamma_{n}\right),
$$

we find that the first two nonzero terms are:

$$
\begin{aligned}
& (-1)^{p-i} f_{p}\left(\gamma_{1}, \cdots, \gamma_{p-i-1}, x^{*} \sigma_{p-i}, \sigma_{p+1-i}, \cdots, \sigma_{n-i}, \gamma_{n-i+1}, \cdots, \gamma_{n}\right) \\
& \quad+(-1)^{p-i+1} f_{p}\left(\gamma_{1}, \cdots, \gamma_{p-i-1}, x^{*}, \sigma_{p-i} \sigma_{p+1-i}, \cdots, \sigma_{n-i}, \gamma_{n-i+1}, \cdots, \gamma_{n}\right) .
\end{aligned}
$$

Now note that $d f_{p}=d f \in A_{j}^{*} \cap A^{n+1}$. Hence, since $i<j$, the above value of $d f_{p}$ is zero. Furthermore, it is clear from the definition of $h_{p}$ and the coboundary formula that the terms of $d h_{p}$ which we have not yet considered above are the same as the remaining terms of $d f_{p}$, except that they carry opposite signs. Hence we have

$$
\begin{aligned}
& d h_{p}\left(\gamma_{1}, \cdots, \gamma_{p-i-1}, x^{*} \sigma_{p-i}, \sigma_{p+1-i}, \cdots, \sigma_{n-i}, \gamma_{n-i+1}, \cdots, \gamma_{n}\right) \\
& \quad=(-1)^{p-i} f_{p}\left(\gamma_{1}, \cdots, \gamma_{p-i-1}, x^{*} \sigma_{p-i}, \cdots, \sigma_{n-i}, \gamma_{n-i+1}, \cdots, \gamma_{n}\right) .
\end{aligned}
$$

Put $g_{p+1}=g_{p}+(-1)^{p-i} h_{p}$. Then $g_{p+1} \in A_{j} \cap A_{i}^{*} \cap A^{n-1}$, and

$$
\left(f-d g_{p+1}\right)\left(\gamma_{1}, \cdots, \gamma_{p-i}, \sigma_{p+1-i}, \cdots, \sigma_{n-i}, \gamma_{n-i+1}, \cdots, \gamma_{n}\right)=0 .
$$

If $p+1<n$, we repeat this construction for $p+1$ instead of $p$, and so continue until we obtain $g_{n} \in A_{j} \cap A_{i}^{*} \cap A^{n-1}$ such that

$$
\left(f-d g_{n}\right)\left(\gamma_{1}, \cdots, \gamma_{n-i-1}, \sigma_{n-i}, \gamma_{n-i+1}, \cdots, \gamma_{n}\right)=0 .
$$

Now consider $\left(f-d g_{n}\right)\left(\gamma_{1}, \cdots, \gamma_{n-i-1}, x^{*} \sigma_{n-i}, \quad \gamma_{n-i+1}, \cdots, \gamma_{n}\right)$. Since $d\left(f-d g_{n}\right)=d f \in A_{j}^{*}$, we have

$$
d\left(f-d g_{n}\right)\left(\gamma_{1}, \cdots, \gamma_{n-i-1}, x^{*}, \sigma_{n-i}, \gamma_{n-i+1}, \cdots, \gamma_{n}\right)=0,
$$

and if this is written out in full according to the coboundary formula we find, using that $f-d g_{n} \in A_{i}^{*}$ and the above, that 


$$
\begin{aligned}
& \left(f-d g_{n}\right)\left(\gamma_{1}, \cdots, \gamma_{n-i-1}, x^{*} \sigma_{n-i}, \gamma_{n-i+1}, \cdots, \gamma_{n}\right) \\
& \quad=\left(f-d g_{n}\right)\left(\gamma_{1}, \cdots, \gamma_{n-i-1}, x^{*}, \gamma_{n-i+1}, \cdots, \gamma_{n}\right) .
\end{aligned}
$$

Thus $f-d g_{n} \in A_{j} \cap A_{i+1}^{*}$, and Proposition 1 is proved.

2. The group $E_{1}^{*}$. Let $f \in A_{j}^{*} \cap A^{i+i}$, and denote by ${ }_{j} f=r_{j}(f)$ the element of $C^{j}\left(G / K, C^{i}(K, M)\right)$ which is obtained by restricting the first $i$ arguments to the invariant subgroup $K$. Thus, if $x \rightarrow x^{*}$ is a choice of representatives in $G$ for the elements of $G / K$, with $K^{*}=1$, we have

$$
{ }_{j} f\left(x_{1}, \cdots, x_{j}\right)\left(\sigma_{1}, \cdots, \sigma_{i}\right)=f\left(\sigma_{1}, \cdots, \sigma_{i}, x_{1}^{*}, \cdots, x_{j}^{*}\right),
$$

and it is clear that ${ }_{j} f$ is actually independent of the particular choice of the representatives $x^{*}$. Evidently, $r_{j}$ induces a homomorphism of $A_{j}^{*} / A_{j+1}^{*}$ onto $C^{i}\left(G / K, C^{i}(K, M)\right)$. Furthermore, it is seen immediately from the coboundary formula and the definition of $A_{j}^{*}$ that, for any $f \in A_{j}^{*}$, we have ${ }_{j}(d f)\left(x_{1}, \cdots, x_{j}\right)=d\left({ }_{j} f\left(x_{1}, \cdots, x_{j}\right)\right)$, i.e., in a more suggestive notation, $r_{j} \circ d=d_{K} \circ r_{j}$, where $d_{K}$ is the coboundary operator for cochains of $K$ in $M$. Hence it is clear that $r_{j}$ induces a homomorphism of $E_{1}^{* j, i}=H^{i+j}\left(A_{j}^{*} / A_{j+1}^{*}\right)$ into $C^{j}\left(G / K, H^{i}(K, M)\right)$. Actually, we shall prove the following:

THEOREM 1. The homomorphism of $E_{1}^{* j, i}$ into $C^{j}\left(G / K, H^{i}(K, M)\right)$ which is induced by the restriction homomorphism $r_{j}: A_{j}^{*} \rightarrow C^{j}\left(G / K, C^{i}(K, M)\right)$ is an isomorphism onto.

We show first that this homomorphism is an isomorphism. Let $f \in$ $A_{j}^{*} \cap A^{i+j+1}$, and suppose that $d f \in A_{j+1}^{*}$ and ${ }_{j} f\left(x_{1}, \cdots, x_{j}\right)=d\left(u\left(x_{1}, \cdots, x_{j}\right)\right)$, where $u \in C^{i}\left(G / K, C^{i}(K, M)\right)$. We have to show that there exists $h \in$ $A_{j}^{*} \cap A^{i+j}$ such that $f-d h \in A_{j+1}^{*}$. Here we have replaced $i$ by $i+1$ for greater convenience in the formulas below. The case $i=0$ (which is thereby omitted) is trivial, since then $f={ }_{j} f$.

Define, for $\sigma_{1}, \cdots, \sigma_{i}$ in $K$ and $\gamma_{1}, \cdots, \gamma_{j}$ in $G, g\left(\sigma_{1}, \cdots, \sigma_{i}, \gamma_{1}, \cdots, \gamma_{j}\right)$ $=u\left(x_{1}, \cdots, x_{j}\right)\left(\sigma_{1}, \cdots, \sigma_{i}\right)$, where $x_{r}=\gamma_{r} K$. If $i=0$ (which is now the case $i=1$ of the theorem), we obtain, since $d f\left(x^{*}, \sigma, \gamma_{1}, \cdots, \gamma_{j}\right)=0$, for $\sigma \in K$,

$$
\begin{aligned}
f\left(x^{*} \sigma, \gamma_{1}, \cdots, \gamma_{j}\right)= & x^{*} \cdot f\left(\sigma, \gamma_{1}, \cdots, \gamma_{j}\right)+f\left(x^{*}, \gamma_{1}, \cdots, \gamma_{j}\right) \\
= & x^{*} \sigma \cdot g\left(\gamma_{1}, \cdots, \gamma_{j}\right)-x^{*} \cdot g\left(\gamma_{1}, \cdots, \gamma_{j}\right) \\
& +f\left(x^{*}, \gamma_{1}, \cdots, \gamma_{j}\right) .
\end{aligned}
$$

The last expression differs from $d g\left(x^{*} \sigma, \gamma_{1}, \cdots, \gamma_{j}\right)$ only by terms whose values are independent of $\sigma \in K$. Hence the value $(f-d g)\left(x^{*} \sigma, \gamma_{1}, \cdots, \gamma_{j}\right)$ is independent of $\sigma$, whence it is clear that $f-d g \in A_{j+1}^{*}$, so that we may take $h=g$ if $i=0$.

If $i>0$, we define a sequence of extensions $g_{1}, \cdots, g_{i}$ of $g=g_{0}$ as follows: the function $g_{k}$ will be defined on the set of $(i+j)$-tuples in which the first $k$ elements and the last $j$ elements are arbitrary elements $\rho_{1}, \cdots, \rho_{k}$ and 
$\gamma_{1}, \cdots, \gamma_{j}$ of $G$, while the remaining elements $\sigma_{r}$ belong to $K$. For the construction which follows we shall use the abbreviation $\gamma_{r}^{s}$ for the $(s-r+1)$ tuple $\left(\gamma_{r}, \gamma_{r+1}, \cdots, \gamma_{s}\right)$, etc. We define the $g_{k}$ 's recursively by the formulas: $g_{1}\left(x^{*} \sigma_{1}, \sigma_{2}^{j}, \gamma_{1}^{j}\right)=x^{*} \cdot g\left(\sigma_{1}^{i}, \gamma_{1}^{j}\right)-f\left(x^{*}, \sigma_{1}^{i}, \gamma_{1}^{j}\right) ; g_{k}\left(\rho_{1}^{k-1}, x^{*} \sigma_{k}, \sigma_{k+1}^{4}, \gamma_{1}^{j}\right)$ $=g_{k-1}\left(\rho_{1}^{k-2}, \rho_{k-1} x^{*}, \sigma_{k}^{j}, \gamma_{1}^{j}\right)+(-1)^{k} f\left(\rho_{1}^{k-1}, x^{*}, \sigma_{k}^{j}, \gamma_{1}^{j}\right)$, for $k>1$. For $k \geqq 1$, we have then $g_{k}\left(\rho_{1}^{k-1}, \sigma_{k}^{j}, \gamma_{1}^{\}}\right)=g_{k-1}\left(\rho_{1}^{k-1}, \sigma_{k}^{i}, \gamma_{1}^{j}\right)$, i.e., each $g_{k}$ is indeed an extension of $g_{k-1}$. Hence we have also $d g_{k}\left(\rho_{1}^{k-1}, \sigma_{k}^{i+1}, \gamma_{1}^{j}\right)=d g_{k-1}\left(\rho_{1}^{k-1}, \sigma_{k}^{i+1}, \gamma_{1}^{j}\right)$. From the first of these relations and from our definition, it follows that, for $1 \leqq l \leqq k$, $g_{k}\left(\rho_{1}^{l-1}, x^{*}, \sigma_{l+1}^{i}, \gamma_{1}^{j}\right)=g_{l}\left(\rho_{1}^{l-1}, x^{*}, \sigma_{l+1}^{j}, \gamma_{1}^{j}\right)=0$.

Now it follows from these facts and the coboundary formula that

$$
\begin{aligned}
d g_{k}\left(\rho_{1}^{k-1}, x^{*}, \sigma_{k}^{i}, \gamma_{1}^{j}\right)= & (-1)^{k} g_{k}\left(\rho_{1}^{k-1}, x^{*} \sigma_{k}, \sigma_{k+1}^{i}, \gamma_{1}^{j}\right) \\
& +(-1)^{k-1} g_{k}\left(\rho_{1}^{k-2}, \rho_{k-1} x^{*}, \sigma_{k}^{i}, \gamma_{1}^{j}\right) \\
= & f\left(\rho_{1}^{k-1}, x^{*}, \sigma_{k}^{i}, \gamma_{1}^{j}\right),
\end{aligned}
$$

for $k>1$.

Also, $d g_{1}\left(x^{*}, \sigma_{1}^{i}, \gamma_{1}^{j}\right)=x^{*} \cdot g_{1}\left(\sigma_{1}^{i}, \gamma_{1}^{j}\right)-g_{1}\left(x^{*} \sigma_{1}, \sigma_{2}^{i}, \gamma_{1}^{j}\right)=f\left(x^{*}, \sigma_{1}^{3}, \gamma_{1}^{j}\right)$. Thus for all $k \geqq 1,\left(f-d g_{k}\right)\left(\rho_{1}^{k-1}, x^{*}, \sigma_{k}^{*}, \gamma_{1}^{3}\right)=0$. We shall show next that the same relation holds with $x^{*} \sigma$ in the place of $x^{*}$.

We have $\left(f-d g_{0}\right)\left(\sigma, \sigma_{1}^{s}, \gamma_{1}^{\}}\right)=0$, from the definition of $g_{0}=g$. Assume that we have already shown that $\left(f-d g_{k-1}\right)\left(\rho_{1}^{k-1}, \sigma, \sigma_{k}^{i}, \gamma_{1}^{j}\right)=0$. Since $d\left(f-d g_{k}\right)\left(\rho_{1}^{k-1}, x^{*}, \sigma, \quad \sigma_{k}^{k}, \gamma_{1}^{j}\right)=0$, we can write the expression $\left(f-d g_{k}\right)\left(\rho_{1}^{k-1}, x^{*} \sigma, \sigma_{k}^{*}, \gamma_{1}^{j}\right)$ as a sum of values of $\pm\left(f-d g_{k}\right)$ for arguments in which the $k$ th place is occupied either by $x^{*}$ or by $\sigma$. The terms in which $x^{*}$ is in the $k$ th place are 0 by what we have just seen. The terms with $\sigma$ in the $k$ th place coincide with the terms obtained by replacing $g_{k}$ with $g_{k-1}$, and are 0 by our inductive assumption. Hence we have $\left(f-d g_{k}\right)\left(\rho_{1}^{k}, \sigma_{k}^{i}, \gamma_{1}^{j}\right)=0$, for all $k \geqq 1$. In particular, for $k=i$, we have $\left(f-d g_{i}\right)\left(\rho_{1}^{i}, \sigma, \gamma_{1}^{j}\right)=0$. Hence, proceeding as just above, if we write $\left(f-d g_{i}\right)\left(\rho_{1}^{i}, x^{*} \sigma, \gamma_{1}^{j}\right)$ as a sum of values of $\pm\left(f-d g_{i}\right)$, with $x^{*}$ and $\sigma$ separated in the argument, we find that the nonzero terms have $x^{*}$ in the $(i+1)$ th place, and are independent of $\sigma \in K$, because $f-d g_{i} \in A_{j}^{*}$. Hence $\left(f-d g_{i}\right)\left(\rho_{1}^{i}, x^{*} \sigma, \gamma_{1}^{j}\right)$ is independent of $\sigma$, whence $f-d g_{i} \in A_{j+1}^{*}$. Thus we may take $h=g_{i}$, and conclude that the homomorphism of Theorem 1 is an isomorphism.

In order to prove that it is onto, we must show that for any $u \in C^{i}\left(G / K, Z^{i}(K, M)\right)$, where $Z^{i}(K, M)$ is the group of the $i$-cocycles for $K$ in $M$, there is an element $h \in A_{j}^{*} \cap A^{i+j}$ such that $d h \in A_{j+1}^{*}$ and ${ }_{j} h=u$.

Define $g \in C^{i}\left(G, Z^{i}(K, M)\right)$ by setting $g\left(\sigma_{1}, \cdots, \sigma_{i}, \gamma_{1}, \cdots, \gamma_{j}\right)$ $=u\left(x_{1}, \cdots, x_{j}\right)\left(\sigma_{1}, \cdots, \sigma_{i}\right)$. If $i=0$ we may evidently take $h=g$. Hence we may suppose that $i>0$. Now we apply exactly the same construction of extensions $g_{1}, \cdots, g_{i}$ of $g$ as in the first part of this proof, where now we take $f=0$. We thus obtain an extension $g_{i}$ of $g$ such that $g_{i} \in A_{j}^{*} \cap A^{i+j}$ and $d g_{i}$ $\in A_{j+1}^{*}$. Clearly, the cochain $h=g_{i}$ satisfies our requirements, and Theorem 1 is proved. 
3. A general identity. We wish to prove a certain identity involving partial coboundary operators which will serve in our subsequent discussion of the differential operator $d_{1}$ of the spectral sequence, and of cup products $\left({ }^{9}\right)$.

Let $f \in A^{i+j-1}, i>0, j>0$. Denote $(i+j)$-tuples of elements of $G$ by $\left(\alpha_{1}, \cdots, \alpha_{i}, \beta_{1}, \cdots, \beta_{j}\right)$. Define the two partial coboundary operators $\delta_{i}$ and $\partial_{j}$ by the formulas:

$$
\begin{aligned}
\delta_{i} f\left(\alpha_{1}, \cdots, \alpha_{i}, \beta_{1}, \cdots,\right. & \left.\beta_{j}\right) \\
= & \alpha_{1} \cdot f\left(\alpha_{2}, \cdots, \alpha_{i}, \beta_{1}, \cdots, \beta_{j}\right) \\
& +\sum_{k=1}^{i-1}(-1)^{k} f\left(\alpha_{1}, \cdots, \alpha_{k} \alpha_{k+1}, \cdots, \alpha_{i}, \beta_{1}, \cdots, \beta_{j}\right) \\
& +(-1)^{i} f\left(\alpha_{1}, \cdots, \alpha_{i-1}, \beta_{1}, \cdots, \beta_{j}\right),
\end{aligned}
$$

and

$$
\begin{aligned}
\partial_{j} f\left(\alpha_{1}, \cdots, \alpha_{i}, \beta_{1}, \cdots,\right. & \left.\beta_{j}\right) \\
= & \beta_{1} \cdot f\left(\beta_{1}^{-1} \alpha_{1} \beta_{1}, \cdots, \beta_{1}^{-1} \alpha_{i} \beta_{1}, \beta_{2}, \cdots, \beta_{j}\right) \\
& +\sum_{k=1}^{j-1}(-1)^{k} f\left(\alpha_{1}, \cdots, \alpha_{i}, \beta_{1}, \cdots, \beta_{k} \beta_{k+1}, \cdots, \beta_{j}\right) \\
& +(-1)^{j} f\left(\alpha_{1}, \cdots, \alpha_{i}, \beta_{1}, \cdots, \beta_{j-1}\right) .
\end{aligned}
$$

Let $S=\left(s_{1}, \cdots, s_{j}\right)$ be an ordered subset of the set $(1,2, \cdots, i+j)$, and denote by $S^{*}=\left(s_{1}^{*}, \cdots, s_{i}^{*}\right)$ its ordered complement. Set $b_{0}=1, b_{k}$ $=\beta_{1} \cdots \beta_{k}$, for $1 \leqq k \leqq j$. For $1 \leqq p \leqq i$, write $p^{*}=s_{p}^{*}-p$ (which is the number of indices $s_{q}<s_{p}^{*}$ ) and set $\nu(S)=\sum_{p=1}^{i} p^{*}$. We define, for any $g \in A^{i+j}$, $g_{s}\left(\alpha_{1}, \cdots, \alpha_{i}, \beta_{1}, \cdots, \beta_{j}\right)=g\left(\gamma_{1}, \cdots, \gamma_{i+j}\right)$, where $\gamma_{s_{q}}=\beta_{g}$ and $\gamma_{s_{p}^{*}}$ $=b_{p^{*}}^{-1} \alpha_{p} b_{p^{*}}$. Finally, we set $g_{j}=\sum_{s}(-1)^{\nu(S)} g_{S}$, where $S$ ranges over all the ordered subsets of $j$ elements from $(1, \cdots, i+j)\left({ }^{10}\right)$. In these terms, we shall establish the following identity:

Proposition 2. For $f \in A^{i+j-1}$, we have

$$
(d f)_{j}=\delta_{i}\left(f_{j}\right)+(-1)^{i} \partial_{j}\left(f_{j-1}\right) .
$$

We consider the terms which occur on the left-hand side of the proposed identity by writing it out in full according to the definition of $(d f)_{j}$ and the coboundary formula. Each coboundary $(d f)_{S}\left(\alpha_{1}, \cdots, \alpha_{i}, \beta_{1}, \cdots, \beta_{j}\right)$ gives

(9) This paragraph, being concerned only with a single group $G$, is independent of the preceding ones. The "shuffling" mechanism which we employ here is closely related to that used by Eilenberg-MacLane in a paper forthcoming in the Ann. of Math. Cf. also Proc. Nat. Acad. Sci. U.S.A. vol. 36 (1950) pp. 657-663.

(10) For instance, with $i=1$ and $j=2$, we have: $g_{2}\left(\alpha_{1}, \beta_{1}, \beta_{2}\right)=g\left(\alpha_{1}, \beta_{1}, \beta_{2}\right)-g\left(\beta_{1}, \beta_{1}^{-1} \alpha_{1} \beta_{1}, \beta_{2}\right)$ $+g\left(\beta_{1}, \beta_{2},\left(\beta_{1} \beta_{2}\right)^{-1} \alpha_{1}\left(\beta_{1} \beta_{2}\right)\right)$, and it will be convenient for the reader to follow the proof of Proposition 2 with this example. 
rise to two types of terms; the "pure" terms in whose arguments each entry has one of the forms $b_{p^{*}}^{-1} \alpha_{p} b_{p^{*}}$, or $b_{p^{*}}^{-1} \alpha_{p} \alpha_{p+1} b_{p^{*}}$, or $\beta_{q}$, or $\beta_{q} \beta_{q+1}$; and the "impure" terms in whose arguments exactly one entry fails to be of this form but is either $b_{p^{*}}^{-1} \alpha_{p} b_{p^{*}} \beta_{p^{*}+1}$ or $\beta_{p^{*}} \cdot b_{p^{*}}^{-1} \alpha_{p} b_{p^{*}}$. Now it is not difficult to see that each impure terms occurs exactly twice, and with opposite signs. In fact, an impure term in which the exceptional entry is of the first form occurs a second time, with the exceptional entry of the second form, for the set $T$ which is obtained from $S$ by switching $s_{p^{*}+1}$ with $s_{p}^{*}$, and since $\nu(T)=\nu(S)+1$, these two terms cancel out. Hence we may conclude that all the impure terms cancel out.

On the other hand, it is clear that the pure terms of the left-hand side of the proposed identity are in one to one correspondence with the terms of the right-hand side. There remains only to verify that they carry the same signs on the two sides. This is easily seen to be the case for the first and the last terms of the coboundaries.

There remains to consider the middle terms. These can be divided into two types, as follows:

(A): The argument contains $i$ elements $b_{p^{*}}^{-1} \alpha_{p} b_{p^{*}}$ and one $\beta_{q} \beta_{q+1}$.

(B): The argument contains $i-1$ elements $b_{p^{*}}^{-1} \alpha_{p} b_{p^{*}}$ and one $b_{p^{*}}^{-1} \alpha_{p} \alpha_{p+1} b_{p^{*}}$.

A term of type (A) occurs on the left with the sign $(-1)^{\nu(S)+s_{q}}$, and occurs on the right with the sign $(-1)^{\nu(T)+i+q}$, where $T$ is the set for which the arguments appear in the same order in the relevant term of $\partial_{j}\left(f_{T}\right)$ as in the relevant term of $(d f)_{S}$. It is easily seen that $\nu(S)-\nu(T)$ is the contribution to $\nu(S)$ which is due to the precedence of $\beta_{g}$ before $\alpha$ 's, because this occurs twice in computing $\nu(S)$ (a second time as the contribution due to the precedence of $\beta_{q+1}$ before the same $\alpha$ 's) but only once in computing $\nu(T)$. Hence $\nu(S)$ $-\nu(T)$ is equal to the number of $s_{p}^{*}$ which are greater than $s_{g}$, i.e., $\nu(S)-\nu(T)$ $=i-\left(s_{q}-q\right)$. Hence the signs for the terms of type (A) are the same on the right as on the left.

Similarly, a term of type (B) occurs on the left with the sign $(-1)^{\nu(S)+\dot{s}_{p}}$ and occurs on the right with the sign $(-1)^{\nu(U)+p}$, where $U$ is the set for which the arguments appear in the same order in the relevant term of $\delta_{i}\left(f_{U}\right)$ as in the relevant term of $(d f)_{s}$. Here we find by an argument quite similar to the above that $\nu(S)-\nu(T)=p^{*}=s_{p}^{*}-p$, whence we see again that the terms of type (B) carry the same signs on the right as on the left. This completes the proof of Proposition 2.

In particular, consider the case $j=1$. Our identity then becomes $(d f)_{1}$ $=\delta_{i}\left(f_{1}\right)+(-1)^{i} \partial_{1}(f)$. If $d f=0$, this reduces to $\partial_{1}(f)=(-1)^{i-1} \delta_{i}\left(f_{1}\right)$, or $(\beta \cdot f)-f$ $=d\left(f_{\beta}\right)$, where $f_{\beta}\left(\alpha_{1}, \cdots, \alpha_{i-2}\right)=(-1)^{i-1} f_{1}\left(\alpha_{1}, \cdots, \alpha_{i-2}, \beta\right)$. This shows again that $G$ operates trivially on $H(G, M)$.

4. The operator $d_{1}$ of the spectral sequence. Let the map $f \rightarrow f_{j}$ be defined as in the last paragraph. Suppose $f \in A_{j-1}^{*} \cap A^{i+j-1}$, and $d f \in A_{j}^{*}$. Let $\beta_{1}, \cdots, \beta_{j}$ be elements of $G$, and write $x_{q}=\beta_{q} K$, where $K$ is the given invariant subgroup 
of $G$. Let $r_{j}$ be the restriction homomorphism of $A_{j}^{*}$ onto $C^{j}(G / K, C(K, M))$, as in $\S 2$. It is seen directly from the definitions that if $g \in A_{j}^{*} \cap A^{i+j}$, the restriction of the first $i$ arguments in $g_{j}$ to $K$ yields the natural image in $C^{i}\left(G, C^{i}(K, M)\right)$ of $r_{j}(g)$. Hence, if, in the identity of Proposition 2 for the above $f$, we restrict $\alpha_{1}, \cdots, \alpha_{i}$ to $K$, we obtain:

$$
r_{j}(d f)\left(x_{1}, \cdots, x_{j}\right)=d\left(h\left(\beta_{1}, \cdots, \beta_{j}\right)\right)+(-1)^{i} d\left(r_{j-1}(f)\right)\left(x_{1}, \cdots, x_{j}\right),
$$

where $h\left(\beta_{1}, \cdots, \beta_{j}\right) \in C^{i-1}(K, M)$ is given by

$$
h\left(\beta_{1}, \cdots, \beta_{j}\right)\left(\alpha_{1}, \cdots, \alpha_{i-1}\right)=f_{j}\left(\alpha_{1}, \cdots, \alpha_{i-1}, \beta_{1}, \cdots, \beta_{j}\right) .
$$

This shows immediately that, if $e$ is the element of $E_{1}^{* j-1, t}$ which corresponds to $f$, and $\phi$ is the isomorphism (Theorem 1) of $E_{1}^{*}$ onto $C(G / K, H(K, M)$ ) which is induced by the maps $r_{j}$, then $\phi\left(d_{1}(e)\right)=(-1)^{i} d(\phi(e))$. Hence we have the following result:

TheOREM 2. Let $\phi$ be the isomorphism of $E_{1}^{*}$ onto $C(G / K, H(K, M))$ which is induced by the restriction homomorphisms $r_{j}$ of $A_{j}^{*}$ onto $C^{j}(G / K, C(K, M))$. Then, for every $e \in E_{1}^{* j, 4}, \phi\left(d_{1}(e)\right)=(-1)^{i} d(\phi(e))$. Hence $\phi$ induces an isomorphism of $E_{2}^{* j, 4}$ onto $H^{j}\left(G / K, H^{i}(K, M)\right)$.

5. The group $E_{1}$, and cup products. By Proposition 1 of $\$ 1$, we know that the injections $A_{j}^{*} \rightarrow A_{j}$ induce an isomorphism, $\psi$, of $E_{1}^{*}$ onto $E_{1}$, which evidently commutes with the operator $d_{1}$. Hence we have also isomorphisms $E_{1} \approx C(G / K, H(K, M))$ and $E_{2} \approx H(G / K, H(K, M))$. In order to be in a position to deal adequately with cup products, we shall investigate the isomorphism of $E_{1}$ onto $C(G / K, H(K, M))$ in greater detail.

An element $e \in E_{1}^{j, i}$ is represented by an element $f \in A_{j} \cap A^{i+j}$ such that $d f \in A_{j+1}$. In the notation of $\S 3$, we have then also $(d f)_{j} \in A_{j+1}$, and $f_{j-1} \in A_{j}$. Hence, if we apply the identity of Proposition 2 to $f$, and restrict the first $i+1$ arguments to $K$, we find that $\delta_{i+1}\left(f_{j}\right)\left(\sigma_{1}, \cdots, \sigma_{i+1}, \gamma_{1}, \cdots, \gamma_{j}\right)=0$. This means that if $f_{j}^{\prime} \in C^{j}\left(G, C^{i}(K, M)\right)$ is defined by $f_{j}^{\prime}\left(\gamma_{1}, \cdots, \gamma_{j}\right)\left(\sigma_{1}, \cdots, \sigma_{i}\right)$ $=f_{j}\left(\sigma_{1}, \cdots, \sigma_{i}, \gamma_{1}, \cdots, \gamma_{j}\right)$, we have, actually, $f_{j}^{\prime} \in C^{j}\left(G, Z^{i}(K, M)\right)$, where $Z^{i}(K, M)$ denotes the group of $i$-cocycles for $K$ in $M$.

On the other hand, by Proposition 1, there is an element $f^{*} \in A_{j}^{*} \cap A^{i+j}$, such that $f-f^{*} \in A_{j+1}+d\left(A_{j}\right)$ and $d f^{*} \in A_{j+1}^{*}$. The element $\psi^{-1}(e)$ is then the natural image of $f^{*}$ in $E_{1}^{* j, 4}$. Furthermore, if $u \in A_{j+1}$, then $u_{j}^{\prime}=0$, and if $v \in A_{j}$, Proposition 2 shows that $(d v)_{j}^{\prime} \in C^{j}\left(G, d\left(C^{i-1}(K, M)\right)\right)$. Hence $f$ and $f^{*}$ determine the same element of $C^{j}\left(G, H^{i}(K, M)\right)$. This means that $f_{j}^{\prime}$ is a representative cochain for $\phi \psi^{-1}(e)$. We may state this as follows:

Proposition 3. Let $\psi$ denote the canonical isomorphism of $E_{1}^{*}$ onto $E_{1}$. Then the homomorphisms $f \rightarrow f_{j}^{\prime}$ of $A_{j}$ into $C^{j}(G, C(K, M))$ induce the isomorphism $\phi \psi^{-1}$ of $E_{1}$ onto $C(G / K, H(K, M))$.

Now let us consider a pairing of two $G$-modules $M$ and $N$ to a third $G$ - 
module $P$. Let $A, B, C$ denote the cochain groups for $G$ in $M, N, P$, respectively, and let $E_{1}, F_{1}, G_{1}$ denote the corresponding terms of the spectral sequences.

Let $f \in A_{j}^{*} \cap A^{i+j}, d f \in A_{j+1}^{*} ; g \in B_{j^{\prime}}^{*} \cap B^{i^{\prime}+j^{\prime}}, \quad d g \in B_{j^{\prime}+1}^{*}$. Then $f \cup g \in$ $C_{j+j^{\prime}} \cap C^{i+i^{\prime}+j+j^{\prime}}$, and $d(f \cup g) \in C_{j+j^{\prime}+1}$. It is seen at once from the definitions of $\S 3$ that $(f \cup g)_{j+j^{\prime}}^{\prime}$ consists only of a single $(-1)^{(v) S}(f \cup g)^{\prime}$; explicitly:

$(-1)^{i^{\prime} i}(f \cup g)_{j+j^{\prime}}\left(\sigma_{1}, \cdots, \sigma_{i+i^{\prime}}, \gamma_{1}, \cdots, \gamma_{j+j^{\prime}}\right)$

$=f\left(\sigma_{1}, \cdots, \sigma_{i}, \gamma_{1}, \cdots, \gamma_{j}\right) \cup \rho \cdot g\left(\gamma^{-1} \sigma_{i+1} \gamma, \cdots, \gamma^{-1} \sigma_{i+i^{\prime}} \gamma, \gamma_{j+1}, \cdots, \gamma_{j+j^{\prime}}\right)$, where $\gamma=\gamma_{1} \cdots \gamma_{j}$, and $\rho=\sigma_{1} \cdots \sigma_{i} \gamma_{1} \cdots \gamma_{j}$. Hence we have

$(f \cup g)_{j+j^{\prime}}^{\prime}\left(\gamma_{1}, \cdots, \gamma_{j+j^{\prime}}\right)=(-1)^{i^{\prime} j} f_{j}^{\prime}\left(\gamma_{1}, \cdots, \gamma_{j}\right) \cup \gamma \cdot\left(g_{j^{\prime}}^{\prime}\left(\gamma_{j+1}, \cdots, \gamma_{j+j^{\prime}}\right)\right)$, or: $(f \cup g)_{j+j^{\prime}}^{\prime}=(-1)^{i^{\prime} j} f_{j}^{\prime} \cup g_{j^{\prime}}^{\prime}$. This proves:

TheOREM 3. Let $\rho=\phi \psi^{-1}$ denote the canonical isomorphism of $E_{1}$ (resp. $\left.F_{1}, G_{1}\right)$ onto $C\left(G / K, H(K, M)\right.$ ) (resp. etc.). Let $u \in E_{1}^{j, s}, v \in F_{1}^{f^{\prime, t^{\prime}}}$, so that $u \cup_{v} \in G_{1}^{j+i^{\prime}, i+i^{\prime}}$. Then $\rho\left(u \cup_{v}\right)=(-1)^{i^{\prime} j} \rho(u) \cup \rho(v)$.

We remark, finally, that the definitions of the cup product and $d_{1}$ give the rule $d_{1}(u \cup v)=d_{1}(u) \cup_{v}+(-1)^{i+j} u \cup d_{1}(v)$, and that this provides a check on the sign in the above. Furthermore, these results imply that Theorem 3 holds also for $E_{2}$, mutatis mutandis.

\section{Chapter III. Applications}

1. The spectral sequence. We begin by recalling a few general facts concerning the spectral sequence. If $Z_{r}^{j}$ denotes the subgroup of $A_{j}$ consisting of all elements $a \in A_{j}$ for which $d a \in A_{j+r}$, we have $E_{r}^{j}=Z_{r}^{j} /\left(Z_{r-1}^{j+1}+d\left(Z_{r-1}^{j+1-r}\right)\right)$. The differential operator $d_{r}$ is the endomorphism of $E_{r}$ which is induced by $d$. The group $E_{r}^{j, i}$ is the canonical image of $Z_{r}^{j} \cap A^{i+i}$ in $E_{r}^{j}$, and we have $d_{r}\left(E_{r}^{j, i}\right) \subset E_{r}^{j+r, i+1-r}$. Hence $d_{r}\left(E_{r}^{j, l}\right)=(0)$, if $r>i+1$, and $d_{r}\left(E_{r}\right) \cap E_{r}^{j}=(0)$, if $r>j$. In particular, if $r>\max (j, i+1)$, then $E_{r}^{j, i}=E_{\infty}^{j, i}$, which is canonically isomorphic with $H^{i+j}(A)_{j} / H^{i+j}(A)_{j+1}$, where $H(A)_{j}$ denotes the image of $H\left(A_{j}\right)$ in $H(A)$. Generally, $E_{r+1}^{j, i} \approx H^{i, i}\left(E_{r}\right)$.

In our case, $H(A)=H(G, M)$. We have canonical maps: $H^{i}(G, M)$ $\rightarrow E_{\infty}^{0, i} \rightarrow E_{2}^{0, i} \approx H^{i}(K, M)^{G}$. The first map is onto, and its kernel is $H^{i}(G, M)_{1}$. The second map is an isomorphism into, and the composite map is the natural restriction homomorphism $r_{i}: H^{i}(G, M) \rightarrow H^{i}(K, M)^{G}$.

On the other hand, we have canonical maps:

$$
H^{j}\left(G / K, M^{K}\right) \approx E_{2}^{j, 0} \rightarrow E_{\infty}^{j, 0} \rightarrow H^{j}(G, M) .
$$

The first map is onto, the second map is an isomorphism into, its image is $H^{i}(G, M)_{j}$, and the composite map is the natural "lifting homomorphism" $l_{j}: H^{j}\left(G / K, M^{K}\right) \rightarrow H^{i}(G, M)$. 
All these facts are consequences of the general properties of the spectral sequence, combined with the results of Chapter II.

\section{A decomposition theorem.}

THEOREM 1. Let $G$ be a finite group, $K$ an invariant subgroup of $G, m$ $=[G: K], n=[K:(1)]$, and suppose that $m$ and $n$ are relatively prime. Then, for each $j>0, H^{j}(G, M)$ can be decomposed uniquely into a direct sum $U+V$. where $V$ is mapped isomorphically onto $H^{i}(K, M)^{G}$ by the restriction homomorphism $r_{j}$, and where $U$ is the isomorphic image of $H^{j}\left(G / K, M^{K}\right)$ by $l_{j}$. Moreover, this decomposition is multiplicative with respect to cup products (11).

First, let $Q$ be a finite group of order $q, B$ a $Q$-module, $f \in Z^{k}(Q, B)$ with $k>0$. Define $f^{\prime} \in C^{k-1}(Q, B)$ by setting $f^{\prime}\left(\gamma_{1}, \cdots, \gamma_{k-1}\right)=\sum_{\gamma \in Q}$ $f\left(\gamma_{1}, \cdots, \gamma_{k-1}, \gamma\right)$. Then we have $d f^{\prime}=(-1)^{k} q f$. Hence, for any $u \in H^{k}(Q, B)$, $q u=0\left({ }^{12}\right)$.

Hence, in our present situation, if $u \in H^{j}\left(G / K, H^{i}(K, M)\right)$, then $n u=0$ if $i>0$, and $m u=0$ if $j>0$. By the results of Chapter II, the same holds therefore for any $u \in E_{2}^{j, i}$, and hence also for any $u \in E_{r}^{j, i}$, if $r \geqq 2$. In particular, it follows that $E_{r}^{j, i}=(0)$, if $r \geqq 2, i>0$, and $j>0$. Now we have $d_{r}\left(E_{r}^{j, i}\right)$ $\subset E_{r}^{j+r, i+1-r}$. If $r \geqq 2$, we have therefore $d_{r}\left(E_{r}^{j, i}\right)=(0)$, unless $i=r-1$ and $j=0$. But if $e \in E_{r}^{0, r-1}$, then $n d_{r} e=d_{r}(n e)=0$, and also $m d_{r} e=0$, since $d_{r} e \in E_{r}^{r, 0}$. Hence $d_{r}=0$, for all $r \geqq 2$.

Hence $E_{2}^{j, 0} \rightarrow E_{\infty}^{j, 0}$ and $E_{\infty}^{0, j} \rightarrow E_{2}^{0, j}$ are isomorphisms onto and by $\S 1$ this means that $l_{j}: H^{j}\left(G / K, M^{K}\right) \rightarrow H^{j}(G, M)$ is an isomorphism, and $r_{j}: H^{j}(G, M)$ $\rightarrow H^{j}(K, M)^{G}$ is onto. Since $E_{\infty}^{p, q}=(0)$ for $p>0$ and $q>0$, it follows furthermore that $H^{j}(G, M)_{1}=H^{j}(G, M)_{j}$. Since these groups are respectively the kernel of $r_{j}$ and the image of $l_{j}$, the following sequence is exact:

$$
(0) \rightarrow H^{i}\left(G / K, M^{K}\right) \underset{l_{j}}{\rightarrow} H^{j}(G, M) \underset{r_{j}}{\rightarrow} H^{i}(K, M)^{G} \rightarrow(0) .
$$

Now choose integers $a$ and $b$ such that $a m+b n=1$. If $x \in H^{j}(G, M)$, set $\alpha(x)=a m x, \beta(x)=b n x$, so that $x=\alpha(x)+\beta(x)$. We have then $\alpha \beta=\beta \alpha=0$, $\alpha^{2}=\alpha$, and $\beta^{2}=\beta$. Hence $\alpha$ and $\beta$ define a decomposition of $H^{i}(G, M)$, and we claim that this decomposition satisfies the requirements of Theorem 1.

In fact, it is clear that $r_{j} \beta=0$, and-using the exactness of the above sequence - one sees easily that $r_{j}$ maps $\alpha\left(H^{j}(G, M)\right)$ isomorphically onto $H^{j}(K, M)^{\sigma}$, while $l_{j}$ maps $H^{j}\left(G / K, M^{K}\right)$ isomorphically onto $\beta\left(H^{j}(G, M)\right)$.

Now let $u \in H^{j}(G, M)$ and $v \in H^{j^{\prime}}(G, N)$, where $M$ and $N$ are two $G$ modules which are paired to a third $G$-module, $P$. Then we have, clearly,

(11) By means of the transfer homomorphism (of the cohomology group of a subgroup into that for the whole group) which has recently been defined by Eckmann and, independently, by Artin, a very simple proof for Theorem 1 can be given. The proof we give here is to serve as an illustration of the use of the spectral sequence.

(12) This result is, of course, well known. 
$\alpha(u) \cup \alpha(v)=\alpha^{2}(u \cup v)=\alpha(u \cup v)$, and $\beta(u) \cup \beta(v)=\beta^{2}(u \cup v)=\beta(u \cup v)$.

Finally, it is clear that $V$ is uniquely characterized as the subgroup of $H^{i}(G, M)$ consisting of all elements whose orders divide $n$.

3. The transgression. We recall that the transgression is a certain homomorphism which arises from an arbitrary spectral sequence in the following way:

Write $E_{0}^{0}=R$, so that $E_{1}^{0, i}=H^{i}(R)$. Also, let $S^{i}=E_{1}^{i, 0}$ and $S=\sum_{i=1}^{\infty} S^{i}$. Then $d_{1}\left(S^{i}\right) \subset S^{i+1}$, and the corresponding cohomology groups $H^{i}(S)$ are the $E_{2}^{4,0}$. Hence, for $i \geqq 2$, the spectral sequence gives a natural homomorphism $\sigma_{i}$ of $H^{i}(S)$ onto $E_{i}^{, 0,}$. Furthermore, the injection of $Z_{i}^{i}$ into $A_{1}$ induces an isomorphism $\mu_{i}$ of $E_{i}^{i, 0}$ into $H^{i}\left(A_{1}\right)$, if $i \geqq 2$. Clearly the composite map $\mu_{i} \sigma_{i}$ is the homomorphism $\nu_{i}$ of $H^{i}(S)$ into $H^{i}\left(A_{1}\right)$ which is induced by the injection of $S$ into $A_{1}$. Now let $i \geqq 2$, and consider the following diagram:

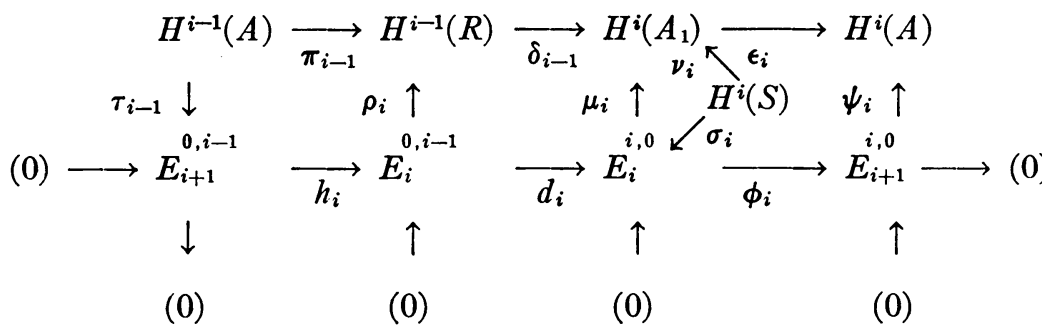

Here, the top line is the natural exact sequence for the pair $\left(A, A_{1}\right)$, noting that $R=A / A_{1}$. The bottom line is composed of natural maps of the spectral sequence, and its exactness is evident from the fact that $E_{i+1} \approx H\left(E_{i}\right)$. The vertical lines are also exact sequences; the nontrivial maps in them are the natural maps induced by injections of subgroups of $A$. Finally, all the commutativity relations are satisfied.

Now an element $x \in H^{i-1}(R)$ is called transgressive if $\delta_{i-1}(x) \in \nu_{i}\left(H^{i}(S)\right)$. If $N_{i}$ denotes the kernel of $\nu_{i}$, then $t_{i}(x)$ is defined as the coset $\nu_{i}^{-1} \delta_{i-1}(x)$ in $H^{i}(S) / N_{i}$. The map $t_{i}$ is called the transgression, and we shall see that, essentially, $t_{i}$ is the map $d_{i}: E_{i}^{0, i-1} \rightarrow E_{i}^{4,0}$; more precisely:

Proposition 1. Let $x \in E_{1}^{0, i-1}$, with $i \geqq 2$. Then $x$ is transgressive if and only if there is an element $y \in E_{i}^{0, i-1}$ such that $x$ is the canonical image $\rho_{i}(y)$ of $y$ in $E_{1}^{0, i-1}$, and then $t_{i}(x)$ is the inverse image $\sigma_{i}^{-1}\left(d_{i} y\right)$ of $d_{i} y$ under the natural homomorphism $\sigma_{i}$ of $E_{2}^{i, 0}$ onto $E_{i}^{i, 0}$.

In fact, if $x=\rho_{i}(y)$, then $\delta_{i-1}(x)=\delta_{i-1} \rho_{i}(y)=\mu_{i} d_{i}(y)$, by the diagram. Since $\sigma_{i}$ is onto, there is a $z \in H^{i}(S)$ such that $\sigma_{i}(z)=d_{i}(y)$. Then $\delta_{i-1}(x)=\mu_{i} \sigma_{i}(z)$ $=\nu_{i}(z)$, showing that $x$ is transgressive. Since the kernel of $\sigma_{i}$ coincides with $N_{i}$, we have then $t_{i}(x)=\sigma_{i}^{-1}\left(d_{i} y\right)$.

On the other hand, if $\delta_{i-1}(x)=\nu_{i}(z)$ (i.e., if $x$ is transgressive) then, by the diagram, $\psi_{i} \phi_{i} \sigma_{i}(z)=\epsilon_{i} \mu_{i} \sigma_{i}(z)=\epsilon_{i} \nu_{i}(z)=\epsilon_{i} \delta_{i-1}(x)=0$, and hence $\phi_{i} \sigma_{i}(z)=0$. Hence there is an element $y_{1} \in E_{i}^{0,1-1}$ such that $d_{i} y_{1}=\sigma_{i}(z)$. Now $\delta_{i-1}(x)$ 
$=\mu_{i}\left(d_{i} y_{1}\right)=\delta_{i-1} \rho_{i}\left(y_{1}\right)$, and hence there is an element $a \in H^{i-1}(A)$ such that $x-\rho_{i}\left(y_{1}\right)=\pi_{i-1}(a)$. Put $y=y_{1}+h_{i} \tau_{i-1}(a)$. Then $\rho_{i}(y)=\rho_{i}\left(y_{1}\right)+\rho_{i} h_{i} \tau_{i-1}(a)$ $=\left(x-\pi_{i-1}(a)\right)+\pi_{i-1}(a)=x$, and Proposition 1 is proved.

In our applications, $A=C(G, M)$, and the restriction of cochains from $G$ to $K$ clearly induces an isomorphism of $R$ onto $C(K, M)$. On the other hand, by Theorem 1 of Chapter II, $S^{i}$ may be identified with $C^{i}\left(G / K, M^{K}\right)$. The above definition now becomes the following: an element $x \in H^{i-1}(K, M)$ is called transgressive if there is a cochain $f \in C^{i-1}(G, M)$ whose restriction to $K$ is a representing cocycle for $x$ and which is such that $d f$ is the natural image in $Z^{i}(G, M)$ of an element of $Z^{i}\left(G / K, M^{K}\right)$. Proposition 1 means that the transgressive elements of $H^{i-1}(K, M)$ make up exactly the canonical image of $E_{i}^{0, i-1}$ in $H^{i-1}(K, M)$, and that $t_{i}$ takes its values in that factor group of $H^{i}\left(G / K, M^{K}\right)$ which is canonically isomorphic with $E_{i}^{x, 0}$. More precisely, if $x$ and $f$ are as above, then $t_{i}(x)$ is the element of this factor group which is determined by $d f$.

\section{An exact sequence involving the transgression.}

Theorem 2. Let $m \geqq 1$, and assume that $H^{n}(K, M)=(0)$, for $0<n<m$. Then the subgroup constituted by the transgressive elements of $H^{m}(K, M)$ coincides with $H^{m}(K, M)^{G}$, the image $t_{m+1}\left(H^{m}(K, M)^{G}\right)$ is a subgroup of $H^{m+1}\left(G / K, M^{K}\right)$, and the following sequence is exact:

$(0) \rightarrow H^{m}\left(G / K, M^{K}\right) \underset{l_{m}}{\rightarrow} H^{m}(G, M) \underset{r_{m}}{\rightarrow} H^{m}(K, M)^{G}$

$$
\underset{t_{m+1}}{\longrightarrow} H^{m+1}\left(G / K, M^{K}\right) \underset{l_{m+1}}{\longrightarrow} H^{m+1}(G, M) .
$$

Since $E_{2}^{1,0}$ is canonically isomorphic with $E_{\infty}^{1,0}$, and so with $H^{1}(G, M)_{1}$, it is clear that $l_{1}$ is an isomorphism. Hence, by induction on $m$, it will suffice to prove the result under the assumption that $l_{m}$ is an isomorphism into. The hypothesis of the theorem gives that $E_{r}^{j, i}=(0)$, for $0<i<m$ and all $r \geqq 2$. Taking $j=m-i$ and $r=m+1$, we conclude from this that $H^{m}(G, M)_{m}$ $=H^{m}(G, M)_{1}$. Thus the image of $l_{m}$ coincides with the kernel of $r_{m}$.

Further, $d_{r}\left(E_{r}^{0, m}\right) \subset E_{r}^{r, m+1-r}=(0)$, if $2 \leqq r \leqq m$. Hence, $E_{2}^{0, m}$ is canonically isomorphic with $E_{m+1}^{0, m}$, which means, by what we have seen in $\S 3$, that the transgressive elements of $H^{m}(K, M)$ are precisely those of $H^{m}(K, M)^{a}$.

We have also $E_{r}^{m+1-r, r-1}=(0)$, if $2 \leqq r \leqq m$, and we may conclude from this that $E_{m+1}^{m+1,0}$ is canonically isomorphic with $E_{2}^{m+1,0}$. Hence the homomorphism $\sigma_{m+1}$ of $\S 3$ is an isomorphism, whence $t_{m+1}$ maps $H^{m}(K, M)^{G}$ onto a subgroup of $H^{m+1}\left(G / K, M^{K}\right)$. Moreover, $t_{m+1}$ corresponds canonically to the map $d_{m+1}: E_{m+1}^{0, m} \rightarrow E_{m+1}^{m+1,0}$. Hence the kernel of $t_{m+1}$ is the canonical image of $E_{m+2}^{0, m}$ in $H^{m}(K, M)^{\sigma}$; but $E_{m+2}^{0, m}$ is canonically isomorphic with $H^{m}(G, M) / H^{m}(G, M)_{1}$, whence we conclude that the kernel of $t_{m+1}$ coincides with the image of $r_{m}$.

Furthermore, the image of $t_{m+1}$ corresponds canonically to $d_{m+1}\left(E_{m+1}^{0, m}\right)$, which is precisely the kernel of the natural homomorphism: $E_{m+1}^{m+1,0} \rightarrow E_{m+2}^{m+1,0}$ 
$\approx H^{m+1}(G, M)_{m+1}$. This means that the image of $t_{m+1}$ coincides with the kernel of $l_{m+1}$, and our proof is complete.

REMARK. In the case $m=1$, the hypothesis of the preceding theorem is vacuous, and hence we have always the following exact sequence:

$(0) \rightarrow H^{1}\left(G / K, M^{K}\right) \rightarrow H^{1}(G, M) \rightarrow H^{1}(K, M)^{G} \rightarrow H^{2}\left(G / K, M^{K}\right) \rightarrow H^{2}(G, M)$.

5. Interpretation in the theory of simple algebras $\left({ }^{13}\right)$. A particularly interesting case of Theorem 2 is the case where $M$ is the multiplicative group $L^{*}$ of a field $L$, and $G$ is a finite group of automorphisms of $L$. Then, as is well known, $H^{1}(K, M)=(0)$. The exact sequence of Theorem 2, for $m=1$,

$$
(0) \rightarrow H^{2}\left(G / K, F^{*}\right) \underset{l_{2}}{\rightarrow} H^{2}\left(G, L^{*}\right) \underset{r_{2}}{\rightarrow} H^{2}\left(K, L^{*}\right)^{G} \underset{t_{3}}{\rightarrow} H^{3}\left(G / K, F^{*}\right) \underset{l_{3}}{\rightarrow} H^{3}\left(G, L^{*}\right)
$$

is then significant for the theory of the simple algebras which have the fixed field, $F$, say, of $K$ in $L$ for center, and which are split by $L\left({ }^{14}\right)$.

Let $U$ be such an algebra. Then there is a vector space $V$ over $L$ which is at the same time a right $U$-module in such a way that $L U^{\prime}$ is the ring of all $L$-linear transformations of $V$, where $U^{\prime}$ denotes the ring of endomorphisms of $V$ which corresponds (by an anti-isomorphism) to $U$. Those nonzero $(L, K)$-semilinear transformations of $V$ which commute with the elements of $U^{\prime}$ are automorphisms, and constitute a group $S$. The map which associates with each $s \in S$ the corresponding automorphism $\sigma$ of $L(s l=\sigma(l) s)$ is a homomorphism $\phi$ of $S$ onto the Galois group $K$ of $L / F$ whose kernel is precisely $L^{*}$. Thus, to each algebra $U$, as above, we obtain a group extension $(S, \phi)$ of $L^{*}$ by $K$. It follows from the theory of simple algebras that this construction $\left({ }^{15}\right)$ establishes an isomorphism of the Brauer group of the algebra classes over $F$ with splitting field $L$ onto the group of extensions of $L^{*}$ by $K$, where the multiplication in the latter is the Baer product. Actually, the commutator ring of $U^{\prime}$ in the full endomorphism ring of $V$ consists of all sums of elements of $S$ and is a crossed product, $L(K, f)$, in the similarity class of $U$, where $f$ is the "factor set," i.e., $f \in Z^{2}\left(K, L^{*}\right)$. Moreover, $f$ is also a factor set belonging to the group extension $(S, \phi)$, and this correspondence gives an isomorphism of the group of extensions of $L^{*}$ by $K$ onto $H^{2}\left(K, L^{*}\right)$.

Now let $T$ denote the fixed field of $G$ in $L ; T \subset F \subset L$. The algebra $U$ is normal over $T$ (in the sense that $T$ coincides with the fixed subring of $U$ for the group of all automorphisms of $U / T$ ) if and only if every automorphism of $F / T$ can be extended to an automorphism of $U$. It is easily seen

${ }^{(13)}$ For the classical theory of simple algebras, see, for instance, Deuring, Algebren, and Artin, Nesbitt, Thrall, Rings with minimum condition.

(14) The exactness of the first half of this sequence is well known, cf. $[9 ; 11]$.

(15) This direct construction of the "crossed product" of a given algebra class is due to J. Dieudonné (La théorie de Galois des anneaux simples et semi-simples, Comment Math. Helv. vol. 21 (1948) pp. 154-184). 
from the above that this is the case if and only if every automorphism of $F / T$ can be extended to an "admissible" automorphism of $(S, \phi)$, i.e., to an automorphism of $S$ which coincides, on $L^{*}$, with a field automorphism of $L$. This, in turn, is easily seen to be the case if and only if the corresponding element of $H^{2}\left(K, L^{*}\right)$ is $G$-fixed. Hence our group $H\left(K, L^{*}\right)^{G}$ is isomorphic with the group of those algebra classes over $F$ whose members are split by $L$, and normal over $T\left({ }^{16}\right)$.

If $U$ is normal over $T$, then the extensions of the elements of $G / K$ to admissible automorphisms of $(S, \phi)$ allow one to regard $S$ as a $G / K$-kernel, in the sense of Eilenberg-MacLane. This means the following:

If $x^{*}$ is an admissible automorphism of $S$ which extends $x \in G / K$, then there are elements $s(x, y) \in S$ such that $x^{*} y^{*}=s(x, y)^{\cdot}(x y)^{*}$, where $s^{*}$ denotes the inner automorphism of $S$ which is effected by $s$. In fact, $x^{*} y^{*}(x y)^{*-1}$ induces on $L^{*}$ an automorphism belonging to $K$. Hence there is an element $s_{1}(x, y)$ in $S$ such that $x^{*} y^{*}(x y)^{*-1}=\psi(x, y) s_{1}(x, y)$, where $\psi(x, y)$ is an automorphism leaving the elements of $L^{*}$ fixed. Using the fact that $H^{1}\left(K, L^{*}\right)$ $=(0)$, one shows that such an automorphism is an inner automorphism effected by an element of $L^{*}$, whence our assertion follows. This defines the structure of a $G / K$-kernel on $S$.

Now one shows that $x^{*}(s(y, z)) s(x, y z)=f(x, y, z) s(x, y) s(x y, z)$, where $f \in Z^{3}\left(G / K, F^{*}\right)$, and that the cohomology class of $f$ (in $H^{3}\left(G / K, F^{*}\right)$ ) does not depend on the particular choice of the extensions $x^{*}$. We choose the $x^{*}$ such that $1^{*}=1$, and denote by $\hat{x}$ the automorphism of $L^{*}$ which is induced by $x^{*}$. Also we choose elements $s_{1}(\sigma) \in S$ such that $\phi\left(s_{1}(\sigma)\right)=\sigma \in K$, taking $s_{1}(1)=1$. Now define, for $\sigma, \tau$ in $K$,

$$
l(\sigma \hat{x}, \tau \hat{y})=s_{1}(\sigma) x^{*}\left(s_{1}(\tau)\right) s(x, y) s_{1}\left(\sigma \hat{x} \tau \hat{y}((\widehat{x y}))^{-1}\right)^{-1} .
$$

Then one can verify directly that each $l(\alpha, \beta)$ commutes with every element of $L^{*}$, and hence belongs to $L^{*}$, i.e., $l \in C^{2}\left(G, L^{*}\right)$. Furthermore, a direct computation shows that $d l(\sigma \hat{x}, \tau \hat{y}, \rho \widehat{z})=f(x, y, z)$. Also, we have $l(\sigma, \tau)$ $=s_{1}(\sigma) s_{1}(\tau) s_{1}(\sigma \tau)^{-1}$, i.e., the restriction of $l$ to $K^{2}$ is in the cohomology class $u \in H^{2}\left(K, L^{*}\right)^{G}$ which is determined by $(S, \phi)$, or by $U$.

The cohomology class in $H^{3}\left(G / K, F^{*}\right)$ which is determined by the above $f$ is the "obstruction" of the $G / K$-kernel $S$ as defined by Eilenberg-MacLane, and, at the same time, the "Teichmüller" class of the normal algebra $U$. What we have just seen shows again that the element $u \in H^{2}\left(K, L^{*}\right)^{G}$ is transgressive, and-furthermore-that the transgression, $t_{3}(u)$, is precisely the Teichmüller class. From Theorem 2, we can now conclude that the Teichmüller classes make up exactly the kernel of the homomorphism $l_{3}: H^{3}\left(G / K, F^{*}\right)$ $\rightarrow H^{3}\left(G, L^{*}\right)$, and that the Teichmüller class of an algebra is 0 if and only if the corresponding cohomology class in $H^{2}\left(K, L^{*}\right)^{G}$ is in the canonical image of $H^{2}\left(G, L^{*}\right)$, which is easily seen to be the case if and only if the given alge-

(16) This is a reformulation of a result of Teichmüller [15]. 
bra belongs to the class of a tensor product $F \otimes_{T} B$, where $B$ is a simple algebra with center $T$. These are the results of Teichmüller, Eilenberg and MacLane, $[15 ; 9]\left({ }^{17}\right)$.

\section{An exact sequence giving the cup product reduction.}

Theorem 3. Let $m \geqq 1$. If $m>1$, assume that $H^{n}(K, M)=(0)$, for $n$ $=2, \cdots, m$. Then there is an exact sequence of homomorphisms:

$$
\begin{aligned}
H^{m}\left(G / K, M^{K}\right) \underset{l_{m}}{\rightarrow} H^{m}(G, M) \underset{r_{m}^{\prime}}{\rightarrow} H^{m-1}\left(G / K, H^{1}(K, M)\right) \\
\qquad \overrightarrow{d_{2}^{\prime}} H^{m+1}\left(G / K, M^{K}\right) \underset{l_{m+1}}{\longrightarrow} H^{m+1}(G, M) .
\end{aligned}
$$

The proof is similar to that of Theorem 2. Our assumption gives that $E_{r}^{y, 4}$ $=(0)$, for $i=2, \cdots, m$ and all $r \geqq 2$. Hence $H^{m}(G, M)_{m-i}=H^{m}(G, M)_{m-i+1}$, for $i=2, \cdots, m$, whence $H^{m}(G, M)=H^{m}(G, M)_{m-1}$. Now $H^{m}(G, M)_{m-1} / H^{m}(G, M)_{m}$ is canonically isomorphic with $E_{m+2}^{m-1,1}$, for $m \geqq 1$, with $E_{m+1}^{m-1,1}$, for $m \geqq 2$, and with $E_{m}^{m-1,1}$, for $m>2$. We wish to prove that it is isomorphic with $E_{3}^{m-1,1}$, for all $m \geqq 1$. From what we have just seen, this will follow if we have shown that $E_{m}^{m-1,1}$ is canonically isomorphic with $E_{3}^{m-1,1}$, for $m>2$. But this follows immediately from the fact that $E_{r}^{m-1-r, r}=(0)$, for $r=3, \cdots, m$. Since this last fact holds also for $r=2$, we find, furthermore, that $E_{3}^{m-1,1}$ is canonically isomorphic with the kernel of $d_{2}$ in $E_{2}^{m-1,1}$. Thus, we have a canonical homomorphism of $H^{m}(G, M)$ into $E_{2}^{m-1,1}$ whose kernel coincides with the image $H^{m}(G, M)_{m}$ of $H^{m}\left(G / K, M^{K}\right)$ under $l_{m}$, and whose image is the kernel of $d_{2}$ in $E_{2}^{m-1,1}$. To this there corresponds a homomorphism $r_{m}^{\prime}$ of $H^{m}(G, M)$ into $H^{m-1}\left(G / K, H^{1}(K, M)\right)$. (This homomorphism $r_{m}^{\prime}$ is induced by restricting the first argument of a suitably selected cocycle, representing the given cohomology class, to $K$.) The kernel of $r_{m}^{\prime}$ is the image of $l_{m}$, and the image of $r_{m}^{\prime}$ is the kernel of the homomorphism $d_{2}^{\prime}$ which corresponds canonically to $d_{2}$.

Finally, the kernel of $l_{m+1}$ is the subgroup of $H^{m+1}\left(G / K, M^{K}\right)$ which corresponds to the kernel of the canonical homomorphism of $E_{2}^{m+1,0}$ into $E_{m+2}^{m+1,0}$. Since $E_{r}^{m+1-r, r-1}=(0)$, for $r=3, \cdots, m+1$, we have $E_{m+2}^{m+1,0} \approx E_{3}^{m+1,0}$, so that the kernel in question is $d_{2}\left(E_{2}^{m-1,1}\right)$. Hence the kernel of $l_{m+1}$ is the image of $d_{2}^{\prime}$. This completes the proof.

When $K$ operates trivially on $M$, so that $M^{K}=M$, we can describe the map $d_{2}^{\prime}$ as a cup product. In this case, $H^{1}(K, M)$ is the group Hom $(K, M)$ of all homomorphisms of $K$ into $M$. Let $K^{\prime}$ denote the commutator subgroup of $K$. The factor group $K / K^{\prime}$ may be regarded as a $G / K$-module in the natural fashion. We can define a pairing of this $G / K$-module $K / K^{\prime}$ with Hom $(K, M)$ to $M$ by setting, for $\sigma^{\prime} \in K / K^{\prime}, \sigma$ a representative of $\sigma^{\prime}$ in $K$, and $f \in \operatorname{Hom}(K, M), \sigma^{\prime} \cup f=f(\sigma)$, which, indeed, is independent of our choice of representatives. This is evidently a pairing, compatible with the $G / K$-module

(17) It is apparent that our argument is not confined to group extensions arising from simple algebras. For instance, it applies to idele classes in class field theory. 
structures. From this we obtain a cup product pairing of $H\left(G / K, K / K^{\prime}\right)$. and $H(G / K$, Hom $(K, M))$ to $H(G / K, M)$. We shall now prove the following:

THEOREM 4. Let $G$ be a group, $K$ an invariant subgroup of $G$ which operates trivially on the $G$-module $M$. Let $d_{2}^{\prime}$ denote the homomorphism of $H^{m-1}(G / K$, Hom $(K, M))$ into $H^{m+1}(G / K, M)$ which corresponds to $d_{2}: E_{2}^{m-1,1}$ $\rightarrow E_{2}^{m+1,0}$. Let $c$ denote the element of $H^{2}\left(G / K, K / K^{\prime}\right)$ which is determined by the group extension,

$$
K / K^{\prime} \rightarrow G / K^{\prime} \rightarrow G / K
$$

Then, for every $u \in H^{m-1}(G / K, \operatorname{Hom}(K, M)), d_{2}^{\prime}(u)=-c \cup u$.

It is easy to see this quite directly, with the filtration $\left(A_{j}^{*}\right)$. We shall, however, give another proof which utilizes Theorem 3 of Chapter II, in order to illustrate the multiplicative features of the spectral sequence.

Let unprimed letters refer to the spectral sequence for $M$, primed letters to the spectral sequence for Hom $(K, M)$, and dotted letters to the spectral sequence for $K / K^{\prime}$. The above pairing of $K / K^{\prime}$ and $\operatorname{Hom}(K, M)$ to $M$ induces a pairing of $E_{r}^{*}$ and $E_{r}^{\prime}$ to $E_{r}$. Let us identify the element $u$ of the theorem with its canonical image in $E_{2}^{m-1,1}$. On the other hand, let $u^{\prime}$ denote the element of $E_{2}^{\prime m-1,0}$ which corresponds to $u,\left(H^{m-1}(G / K\right.$, Hom $(K, M))$ being canonically isomorphic with $E_{2}^{\prime m-1,0}$ also). The natural homomorphism of $K$ onto $K / K^{\prime}$ may be regarded as a $G / K$-fixed one-dimensional cohomology class for $K$ in $K / K^{\prime}$, and hence corresponds canonically to an element $v \in E_{2}^{0,0}$. It is evident that $v \cup u^{\prime}=u$, regarded as an element of $E_{2}^{m-1,1}$. We have $d_{2}(u)=d_{2}(v) \cup u^{\prime}-v \cup d_{2}\left(u^{\prime}\right)$, by the formula of the coboundary for cup products of cochains for $G$ (which represent $v, u^{\prime}$, and $u$ ). But since $u^{\prime}$ $\in E_{2}^{\prime m-1,0}$, we have $d_{2}\left(u^{\prime}\right)=0$. Hence $d_{2}(u)=d_{2}(v) \cup u^{\prime}$. Now let $x \rightarrow x^{*}$ denote a choice of representatives in $G$ for the elements of $G / K$, and let $f$ be the map of $G$ into $K / K^{\prime}$ which sends an element $\sigma x^{*}(\sigma \in K)$ into the coset $\bmod K^{\prime}$ of $\sigma$. Then $f$ is a cochain representing $v$; moreover, it is easily verified that $d f$ is the natural image in $C^{2}\left(G, K / K^{\prime}\right)$ of an element $g \in Z^{2}\left(G / K, K / K^{\prime}\right)$, and that $g$ belongs to the cohomology class of $-c$.

Now if we pass to the cohomology groups by the canonical maps, $d_{2}(u)$ becomes $d_{2}^{\prime}(u), d_{2}(v)$ becomes $-c$, and $u^{\prime}$ becomes $u$. By Theorem 3 of Chapter II, the cup product becomes the cup product of the requisite cohomology groups, and hence we obtain, indeed, $d_{2}^{\prime}(u)=-c \cup u$.

Now suppose that $G$ is a free group. Then $K$ is free, and hence the assumptions of Theorem 3 are satisfied. Since now $H^{m}(G, M)=(0)$, for $m \geqq 2$, we conclude that $d_{2}^{\prime}$ is an isomorphism onto for $m>1$, and is a homomorphism onto, with kernel $r_{1}^{\prime}\left(H^{1}(G, M)\right)$, for $m=1$. If, furthermore, $K$ operates trivially on $M$, we can use Theorem 4 to conclude that the map $u \rightarrow c \cup u$ is an isomorphism of $H^{m-1}(G / K$, Hom $(K, M))$ onto $H^{m+1}(G / K, M)$, if $m>1$. In the case $m=1$, this map is a homomorphism of $\operatorname{Hom}(K, M)^{a}$ 
=Ophom $(K, M)$ onto $H^{2}(G / K, M)$, and the kernel is the group of those operator homomorphisms of $K$ into $M$ (i.e., elements of Hom $(K, M)^{g}$ ) which can be extended to cocycles for $G$ in $M$. This is the cup product reduction theorem of Eilenberg-MacLane [8].

\section{BIBLIOGRAPHY}

1. H. Cartan, Sur la cohomologie des espaces où opère un groupe, C.R. Acad. Sci. Paris vol. 226 (1948) pp. 148-150, 303-305.

2. H. Cartan and S. Eilenberg, Satellites des foncteurs de modules, not yet published.

3. H. Cartan and J. Leray, Relations entre anneaux de cohomologie et groupe de Poincare, Colloque Topologie Algebrique, Paris, 1947, pp. 83-85.

4. B. Eckmann, On complexes over a ring and restricted cohomology groups, Proc. Nat. Acad. Sci. U.S.A. vol. 33 (1947) pp. 275-281.

5. — On infinite complexes with automorphisms, Proc. Nat. Acad. Sci. U.S.A. vol. 33, (1947) pp. 372-376.

6. S. Eilenberg, Homology of spaces with operators. I, Trans. Amer, Math. Soc. vol. 61 (1947) pp. 378-417.

7. - Topological methods in abstract algebra. Cohomology theory of groups, Bull. Amer. Math. Soc. vol. 55 (1949) pp. 3-27.

8. S. Eilenberg and S. MacLane, Cohomology theory in abstract groups. I, Ann. of Math. vol. 48 (1947) pp. 51-78.

9. - Cohomology and Galois theory. I, Normality of algebras and Teichmilller's cocycle, Trans. Amer. Math. Soc. vol. 64 (1948) pp. 1-20.

10. - Homology of spaces wilh operators. II, Trans. Amer. Math. Soc. vol. 65 (1949) pp. 49-99.

11. G. Hochschild, Local class field theory, Ann. of Math. vol. 51 (1950) pp. 331-347. 271-292.

12. R. Lyndon, The cohomology theory of group extensions, Duke Math. J. vol. 15 (1948) pp.

13. J-P. Serre, Cohomologie des extensions de groupes, C.R. Acad. Sci. Paris vol. 231 (1950) pp. $643-646$.

14. - Homologie singulière des espaces fibrés. Applications, Ann. of Math. vol. 54 (1951) pp. 425-505.

15. O. Teichmüller, Über die sogenannie nichtkommutative Galoische Theorie und die Relation, Deutsche Mathematik vol. 5 (1940) pp. 138-149.

Yale University, New Haven, Conn.

Paris, France. 